\title{
Electron Transfer based Ultra-bright Organic Afterglow Nanoprobe for Accurate Molecular Imaging in Mice
}

\section{Youjuan Wang}

Hunan University

Jing Guo

Hunan University

Shiyi Liao

Hunan University

Li Xu

Hunan University

Qian Chen

Soochow University

Guosheng Song

Hunan University

Xiaobing Zhang ( $\boldsymbol{\nabla}$ xbzhang@hnu.edu.cn )

Hunan University https://orcid.org/0000-0002-4010-0028

Weihong Tan

Hunan University https://orcid.org/0000-0002-8066-1524

Article

Keywords: afterglow luminescence, molecular imaging, afterglow nanoprobe (TA-BHQ)

Posted Date: November 30th, 2021

DOI: https://doi.org/10.21203/rs.3.rs-1026824/v1

License: (1) (1) This work is licensed under a Creative Commons Attribution 4.0 International License. Read Full License 


\section{Abstract}

Afterglow luminescence can greatly improve the signal-to-background ratio (SBR) of molecule imaging in living animal owing to the no need of real-time light excitation. However, the relatively low luminescence of afterglow nanoprobe and attenuation of maximum intensity (afterglow photobleaching) usually lead to the insufficient sensitivity and the inaccurate quantification for repeated molecular imaging.

Furthermore, the requirement of high power of light excitation (up to $1 \mathrm{~W} / \mathrm{cm}^{2}$ ) may result in the inevitable phototoxicity, and the long acquisition time (up to $1 \mathrm{~min}$ ) make it difficult to detect the rapid biological events. Herein, we design electron-rich trianthracene derivatives (TA)-based organic afterglow nanoparticles (TA-NPs) for high-sensitive, safe, lossless and longitudinal molecular imaging. Notably, a great enhancement of afterglow luminescence performance over the previous reported afterglow nanoparticles is achieved though electron transfer engineering (Table 1): Specifically, TA-NPs can be excited by room light with ultra-low power $\left(58 \mu \mathrm{W} / \mathrm{cm}^{2}\right)$ and with ultra-short acquisition time $(0.01 \mathrm{~s})$. The luminescent intensity of TA-NPs is 500-fold of commonly used organic MEHPPV-based nanoparticles. Negligible afterglow photobleaching in mice is observed even after re-excitation for more than 15 cycles. Such ultra-bright afterglow enables the deep-tissue imaging (up to $6.0 \mathrm{~cm}$ ) and the ultra-fast afterglow imaging of freely-moving mice in waken state. Moreover, TA-NPs can dynamically and accurately visualize subcutaneous tumor, orthotopic glioma and distinguish the plaque in carotid atherosclerosis. Finally, we develop an afterglow nanoprobe (TA-BHQ), activated only in the presence of Granzyme $\mathrm{B}$, for tracking the time-sensitive Granzyme B activity as a direct way to monitor immunotherapeutic responses.

\section{Introduction}

Molecular imaging technology enables the dynamical, quantitative visualization of specific biochemical activity without trauma in vivo at cellular or molecular level, so as to study biological process, diagnose disease or monitor therapy ${ }^{1-7}$. High sensitivity together with novel methods to reliably quantitate imaging signal are strongly needed for accurate molecular imaging, which should allow not only the detection of molecular events that occur either with a low mass of substance or at low concentration, but also the repeated studies in the same animal to collect longitudinal data and reduce the number of animals and $\operatorname{cost}^{8-11}$. However, there are several intrinsic limitations for current imaging modalities, such as poor sensitivity (for MRI), ionizing radiation (for PET and CT), or strong background signals (for fluorescence imaging $)^{12}$. Interestingly, afterglow luminescence, called long-persistent luminescence, is an intrinsic luminescent process that occurs after the cessation of light excitation. Owing to no requirement of realtime light excitation, afterglow luminescence can eliminate autofluorescence of biological tissues, which enables to greatly improve signal-to-background ratio (SBR), in contrast to fluorescent imaging ${ }^{13-17}$. Thereby, it holds great promise for various biomedical applications in vivo such as imaging orthotopic liver tumors or tiny peritoneal metastatic tumors, mapping lymph node, early detecting drug-induced hepatotoxicity, or afterglow imaging-guided surgery or therapy in living animals ${ }^{18-21}$. 
To date, a few inorganic nanoparticles (e.g., $\mathrm{ZnGa}_{1.995} \mathrm{Cr}_{0.005} \mathrm{O}_{4}$, lanthanide-doped $\mathrm{NaYbF}_{4}$ upconversion nanoparticles) have been developed to produce afterglow luminescence ${ }^{15}, 16$. However, those may be hampered by systemic toxicity concerns related to the potential leakage of heavy metal ions. Compared with inorganic nanoparticles, organic afterglow nanoparticles (e.g., poly[2-methoxy-5-(2-ethylhexyloxy)1,4-phenylenevinylene] (MEHPPV) or its analogs) are more suitable for biomedical applications in terms of biocompatibility and biodegradability ${ }^{13,22-26}$. Unfortunately, current organic afterglow nanoprobes are usually limited by the poor "photo-afterglow conversion performance", the relatively low afterglow luminescent intensity, the requirement of high power density of laser excitation, the long acquisition time, and even the attenuation in maximum intensity (termed "afterglow photobleaching") for repeated imaging. Specifically, for the typical organic afterglow systems (MEHPPV-based nanoparticles), an 808 $\mathrm{nm}$ of laser with power density of as high as $1 \mathrm{~W} / \mathrm{cm}^{2}$ was required to excite adequate afterglow luminescence, which is $\sim 3$ times higher than the conservative limit $\left(\sim 0.33 \mathrm{~W} / \mathrm{cm}^{2}\right.$, the American National Standard for the Safe Use of Lasers) for $808 \mathrm{~nm}$ laser power density setting for human skin exposure ${ }^{13 \text {, }}$ 14, 27. The afterglow imaging conducted at such excessive illumination may lead to phototoxicity and thereby tissue injury. Moreover, the low luminescence intensity ( $0.01 \%-1 \%$ of fluorescent intensity) and long acquisition time (up to $1 \mathrm{~min}$ ) may result in the low level of key biomolecules and rapid biological events to be missed during lengthy and insensitivity imaging ${ }^{28}$. Furthermore, the serious attenuation in maximum intensity was observed from MEHPPV-based nanoparticles (60\% of decrease after 10 -cycle irradiation) ${ }^{13}$. Such "afterglow photobleaching" due to light-induced degradation of luminophore (e.g., phenylenevinylene) can render the failure of accurate quantification for repeated imaging during hours-long experiment. Thence, efforts of afterglow imaging should focus on not only the design of sensitive organic afterglow molecules with high "photo-afterglow conversion effect" that support ultra-bright afterglow luminescence and safe excitation power, but also the development of negligible photobleaching of afterglow allowing for lossless and accurate imaging over repeated irradiation. To date, technical hurdles continue to hamper accurate, safe, high-sensitivity, and longitudinal afterglow imaging in vivo, the reason is that the ultra-bright afterglow luminescent, ultra-low excitation power, as well as well-recharged nanoparticles remains unexplored.

Herein, we introduce the electron-rich anthracene into the afterglow molecule design and synthesize trianthracene derivatives (TA)-based afterglow nanoparticles (TA-NPs) (Fig. 1a). Electron transfer strategy can significantly promote the reactivity of TA molecules with $\mathrm{O}_{2}$, thereby providing sufficient endoperoxides to excite subsequent luminescence, owing to that the generation of afterglow igniter (superoxide radicals, $\mathrm{O}_{2}{ }^{--}$) is not required to surpass the energy barrier between ${ }^{3} \mathrm{O}_{2}$ and ${ }^{1} \mathrm{O}_{2}$. As expected, a 500-fold enhancement of afterglow luminescence intensity is achieved over the commonly used MEHPPV-based organic nanoparticles (Table 1, Supplementary Table 1 and 2), which enable to image targets through deep tissue (up to $6.0 \mathrm{~cm}$ ) and capture the afterglow signals within ultra-short acquisition time (down to $0.01 \mathrm{~s}$ ). Notably, TA-NPs can be excited by the light source with ultra-low power density (58 $\left.\mu \mathrm{W} / \mathrm{cm}^{2}\right)$ that is far below the intensity of direct sunshine $\left(0.13 \mathrm{~W} / \mathrm{cm}^{2}\right)^{29}$, and thereby exhibit the negligible attenuation of maximum intensity in mice even after re-excitation of 15 cycles. Such bright 
afterglow, negligible photobleaching and safe excitation endow TA-NPs to realize rechargeable, accurate and longitudinal in vivo imaging, such as visualizing subcutaneous or orthotopic tumors, distinguishing the plaque in carotid atherosclerosis in living mice, and even ultra-fast afterglow imaging for freely moving mice in waken state. Finally, we demonstrate the feasibility of TA-based nanoprobe (TA-BHQ) for activatable and longitudinal afterglow molecular imaging of immunotherapeutic response, after chemotherapy or radiotherapy in vivo.

\section{Results}

\section{Design of novel afterglow luminescent molecules}

For typical organic afterglow process, the vinyl bonds $(\mathrm{C}=\mathrm{C})$ of afterglow substrate (MEHPPV, DO, SO and HBA, Supplementary Table 3, Supplementary Scheme 1$)$ are oxidized by singlet oxygen $\left({ }^{1} \mathrm{O}_{2}\right)$ under light excitation to form the unstable intermediates (1,2-dioxetane) via cycloaddition reaction, followed by radiative relaxation to emit luminescence ${ }^{13}, 24$. However, the generation of ${ }^{1} \mathrm{O}_{2}$ required the triplet energy of sensitizer to be greater than the energy barrier between ${ }^{3} \mathrm{O}_{2}$ and ${ }^{1} \mathrm{O}_{2}(22.5 \mathrm{kcal} / \mathrm{mol})$, which may be a major obstacle that greatly limits the afterglow performance of those probes ${ }^{30}$. Given the generation of intermediates through electron transfer path is not limited by the triplet energy barrier between ${ }^{3} \mathrm{O}_{2}$ and ${ }^{1} \mathrm{O}_{2}{ }^{30}$, herein we exploit the electron transfer mechanism to boost the production efficacy of intermediates and thereby afterglow intensity. We introduce the electron-rich anthracene into the afterglow molecule design to synthesize trianthracene derivatives (TA), which was substituted with alkoxy groups at orthoand bay-positions to enhance the electro-rich properties. Its isomer dianthraphenanthrene (DAP) was also synthesized as a comparison (Fig. 1a; 2. Supplementary Molecule design, Synthesis and Characterization). Such afterglow luminescence mechanism of TA-NPs involves two complementary types of photooxidation process (i.e., Type I and Type II reaction) (Fig. 1a). Specifically, Type I reaction yields superoxide radicals $\left(\mathrm{O}_{2}{ }^{--}\right)$and radical cations of TA $\left(\mathrm{TA}^{*+}\right)$ through electron transfer, while Type II reaction produces ${ }^{1} \mathrm{O}_{2}$ and TA molecules via energy transfer. We hypothesize that Type I pathway will greatly promote the reactivity of TA molecules with $\mathrm{O}_{2}$, resulting in the formation of sufficient endoperoxides (EPOs), because the generation of $\mathrm{O}_{2}{ }^{-{ }^{-}}$is not hampered by the triplet energy of sensitizer in Type II pathway. Subsequently, those EPOs underwent 0-O bond cleavage into aromatic-carbonyl compounds, and spontaneously generated photons.

In order to construct biocompatible afterglow luminescent nanoparticles, we transformed TA or DAP molecules into water-soluble nanoparticles through one-step nanoprecipitation in the presence of dual polymers (poly (styrene-co-maleic anhydride) (PSMA) and 1,2-distearoyl-sn-glycero-3phosphoethanolamine- N-[methoxy(poly(ethylene glycol))] (DSPE-PEG)) (Fig. 1a, Supplementary Table 4). In particular, transmission electron microscope (TEM) image further revealed that TA-based nanoparticles (TA-NPs) appeared as mono-disperse nanoparticles, with a mean hydrodynamic size of $\sim 40 \mathrm{~nm}$ (Fig. 1b). The hydrodynamic diameters of the nanoparticles measured by dynamic light scattering (DLS) 
were about $60 \mathrm{~nm}$ (Fig. 1b, Supplementary Fig. 1). Figure 1c and 1d unveiled the characteristic absorption bands and the fluorescence emission spectra of TA-NPs and DAP-based nanoparticles (DAP-NPs).

Subsequently, the afterglow luminescence of TA-NPs and DAP-NPs was recorded with the IVIS Lumina XR imaging system under bioluminescence (without excitation) modes to identify the capability of afterglow luminescence. Notably, TA-NPs showed stronger luminescence than DAP-NPs after cessation of white light irradiation (Fig. 1e, f). Besides, the afterglow spectra of TA-NPs were similar to its fluorescence spectra from 600-750 nm (Fig. 1g).

\section{Mechanism of afterglow luminescence}

To study the underlying mechanism governing afterglow luminescence through Type I pathway, we conducted the following research. Firstly, to determine the $\mathrm{O}_{2}{ }^{-}$- generation of TA, electron spin resonance (ESR) spectroscopy was initially carried out, using 5,5-dimethyl-1-pyrroline-N-oxide (DMPO) as spin-trap agent for $\mathrm{O}_{2}{ }^{--}$. As shown in Figure 2a, neither TA in dark nor light irradiation alone had any impact on the ESR signals. In contrast, a characteristic paramagnetic adduct was observed for TA under light irradiation and matched with that of referential $\mathrm{KO}_{2}$ (a well-known $\mathrm{O}_{2}{ }^{\circ-}$ generator), indicating the production of $\mathrm{O}_{2}{ }^{\circ-}$. Besides, upon the addition of superoxide dismutase ( $\mathrm{SOD}, \mathrm{O}_{2}{ }^{--}$scavenger), the ESR amplitude was dramatically reduced to background level, which was ascribed to that SOD can consume $\mathrm{O}_{2}{ }^{*-}$. Next, we characterized the formation of radical cations $\left(\mathrm{TA}^{\cdot+}\right)$ through UV-vis-NIR absorption spectra. $\mathrm{TA}^{\cdot+}$ was formed after TA receiving irradiation, and the absorption spectra of TA and TA ${ }^{*+}$ in $D C M$ were recorded (Fig. 2b). Compared with the neutral TA, TA* exhibited several typical absorption peaks at the nearinfrared region ( 750 to $1350 \mathrm{~nm}$ ) and greatly decreased absorption at $\lambda_{\max }=594 \mathrm{~nm}$. Meanwhile, DAP could also produce the radical cations $\mathrm{DAP}^{\circ+}$ and $\mathrm{O}_{2}{ }^{--}$under irradiation (Supplementary Fig. 2). Interestingly, TA showed lower first oxidation potential ( $-0.15 \mathrm{~V})$ compared with the isomer DAP $(-0.12 \mathrm{~V})$, according to the cyclic voltammogram measurement, indicating that TA was easier to give electrons to form free radical cation (Fig. 2c, d). Next, SOD was added into TA-NPs solutions, to further validate that the enhanced afterglow signal was indeed related to the generated $\mathrm{O}_{2}{ }^{\circ}$, as expected, the afterglow luminescence showed $26.8 \%$ of decrease (Fig. 2e). Furthermore, we have measured ${ }^{1} \mathrm{O}_{2}$ generation from TA-NPs via the fluorescence intensities of SOSG at $535 \mathrm{~nm}$ and found that a considerable amount of ${ }^{1} \mathrm{O}_{2}$ was produced during irradiation of TA-NPs (Fig. 2f). Subsequently, the incubation of ${ }^{1} \mathrm{O}_{2}\left(\mathrm{Na}_{2} \mathrm{MoO}_{4}+\right.$ $\mathrm{H}_{2} \mathrm{O}_{2}$ ) with TA-NPs resulted in higher luminescence intensity of TA-NPs, than that of PBS, $\mathrm{H}_{2} \mathrm{O}_{2}$ or $\mathrm{Na}_{2} \mathrm{MoO}_{4}$ alone (Fig. 2g), which confirmed that ${ }^{1} \mathrm{O}_{2}$ was also responsible for afterglow luminescence via Type II pathway. Figure $2 \mathrm{~h}$ showed that the afterglow of TA-NPs in $\mathrm{O}_{2}$-saturated solutions was 1.9 fold that in $\mathrm{N}_{2}$-saturated solutions, highlighting the key role of $\mathrm{O}_{2}$ in afterglow luminescence.

To further understand the mechanism on EPOs processes, density functional theory (DFT) calculations were applied to predict the relative energies of EPOs intermediates briefly, and gave one of possible reaction paths (Fig. 2i, j). The reaction process of $\mathrm{O}_{2}{ }^{\circ-}$ or ${ }^{1} \mathrm{O}_{2}$ cycloaddition in anthracene units to anthracene-dioxetane intermediate was determined, based on the corresponding intermediate EPOs 
found, confirmed by MALDI-TOF MS measurement (Fig. 2i and Supplementary Fig. 3). When exposed to light and air, TA molecules undergo a serial of cycloaddition with $\mathrm{O}_{2}{ }^{--}$or ${ }^{1} \mathrm{O}_{2}$ through electron transfer (Type I pathway) or energy transfer (Type II pathway) reactions to give various EPOs intermediates (II-VI). Specifically, initial compound TA was first additively oxidized by one $\mathrm{O}_{2}{ }^{\circ-}$ or ${ }^{1} \mathrm{O}_{2}$ molecule, forming the corresponding intermediate EPOs (II or III). Since EPOs (II or III) contained surplus reaction sites in other anthracene units, additional $\mathrm{O}_{2}{ }^{\cdot-}$ or ${ }^{1} \mathrm{O}_{2}$ molecule was added to generate the intermediate EPOs (IV, $\mathrm{V}$ or VI). Given that the energy of EPOs (II and IV) was slightly lower than that of EPOs (III and V) (Fig. 2j), it might be prone to generate EPOs (II and IV) during gradual cycloaddition reaction. After long-term light irradiation of TA, the typical UV-vis absorption bonds (500 - $600 \mathrm{~nm}$ ) and typical fluorescent emission peak at $650 \mathrm{~nm}$ were significantly decreased, indicating the decomposition of chemical structures for TA molecules (Supplementary Fig. 4). Besides, the obvious carbonyl characteristic peak of oxidized TA fragments was detected in Fourier transform infrared spectroscopy (FTIR) at 1,735 cm${ }^{-1}$, which confirmed the oxidation of TA into various aromatic-carbonyl compounds (Fig. 2k).

Based on the above results, we created a possible picture of underlying mechanism for TA-NPs afterglow luminescence. In the photooxidation, TA-NPs underwent two different photochemical reaction pathways to generate EPOs and thereby afterglow luminescence: electron transfer pathway (Type I) and energy transfer pathway (Type II) ${ }^{30,31}$. During the Type I afterglow, TA molecules are firstly excited by light and subsequently transfer an electron to ${ }^{3} \mathrm{O}_{2}$ to produce $\mathrm{O}_{2}{ }^{\cdot-}$ and $\mathrm{TA}^{*+}$. During the Type II afterglow, the triplet state of TA molecules excited by light can transfer energy to ${ }^{3} \mathrm{O}_{2}$ to generate ${ }^{1} \mathrm{O}_{2}$. Next, TA or $\mathrm{TA}^{\cdot+}$ provide reaction sites to trap $\mathrm{O}_{2}{ }^{*-}$ or ${ }^{1} \mathrm{O}_{2}$, resulting in a large amount of EPOs. Because the generation of $\mathrm{O}_{2}{ }^{-\cdots}$ is no longer restricted by the triplet energy of sensitizer, the Type I pathway may greatly increase the reactivity of TA molecules with ${ }^{3} \mathrm{O}_{2}$ for formation of a large amount of EPOs. Finally, intermediate EPOs can undergo 0-0 cleavage and thereby generate afterglow luminescence.

Compared with DAP-NPs, TA-NPs possess three complete anthracene units and showed higher afterglow luminescent intensity, indicating the introduction of electron-rich anthracene units was crucial for enhancing afterglow luminescence. Similarly, DAP-NPs could also produce $\mathrm{O}_{2}{ }^{--}$and ${ }^{1} \mathrm{O}_{2}$ under photooxidation (Supplementary Figs. 2 and 5). Notably, TA-NPs had a lower oxidation potential in contrast to DAP, and were more likely to give electrons to form $\mathrm{TA}^{*+}$ and $\mathrm{O}_{2}{ }^{-*}$, which may increase the path of Type I afterglow reaction (Fig. 2C). We also calculated the process of addition of ${ }^{1} \mathrm{O}_{2}$ to DAP using DFT calculations, and found that the energy of EPOs (II-VI) from TA were lower than those corresponding EPOs from DAP, suggesting the larger possibility of forming EPOs for TA (Supplementary Fig. 6).

\section{Optimization and performance study of afterglow luminescence}

We subsequently investigated the effect of amphiphilic polymers on TA-NPs afterglow luminescence, and found that the dual polymers (PSMA + DSPE-PEG) co-coating resulted in higher afterglow luminescence, in contrast to other kinds of polymer coating groups (e.g., pluronic-F127 or PSMA only) (Supplementary 
Fig. 7). Therefore, the polymers (PSMA + DSPE-PEG) co-coated nanoparticles were used for the subsequent in vitro/vivo experiments.

Currently, the semiconducting polymer (MEHPPV) is the most commonly used organic afterglow material, reported in the previous articles ${ }^{13,14,19}$. We further compared the afterglow performance of TANPs with MEHPPV-based nanoparticles (MEHPPV-NPs) and other organic molecules-based nanoparticles such as anthracene, perylene, naphthalene, respectively (Fig. 3a, Supplementary Table 5). Then, we characterized those nanoparticles including UV-vis absorption spectra, fluorescence emission spectra and DLS sizes (Supplementary Figs. 8 - 11). We found that TA-NPs showed extremely higher afterglow intensity than that of MEHPPV-, anthracene-, perylene-, and naphthalene-based nanoparticles. Especially, the afterglow intensity of TA-NPs was 500-fold that of MEHPPV-NPs (Fig. 3b, c, Supplementary Fig. 12).

Through the continuous acquisition of afterglow images, the long-lasting afterglow luminescence could persist for over 60 mins for TA-NPs after light cessation, with a prolonged half-life of $\sim 16 \mathrm{~min}$ at biologically relevant conditions ( $\mathrm{pH}=7.4$ at $37^{\circ} \mathrm{C}$; Fig. 3d), compared with half-life of $\sim 6 \mathrm{~min}$ for MEHPPV-NPs. Moreover, as the power density of light irradiation increasing from 1.4 to $15 \mathrm{~mW} / \mathrm{cm}^{2}$, TANPs showed gradually brighter afterglow luminescence (Fig. 3f, Supplementary Fig. 13). The afterglow intensities of TA-NPs were continuously enhanced with the irradiation time increasing, and reached the maximum at $8 \mathrm{~s}$ of irradiation $\left(15 \mathrm{~mW} / \mathrm{cm}^{2}\right)$ (Fig. 3g, Supplementary Fig. 14). Furthermore, afterglow luminescence of TA-NPs became brighter as the concentration increasing, and showed a linear correlation between afterglow intensities and concentrations $(0-40 \mu \mathrm{g} / \mathrm{mL})($ Fig. 3h, Supplementary Fig. 15). As a result, under various power density, irradiation time or concentration, TA-NPs showed extremely higher afterglow intensities than that of MEHPPV-NPs (Fig. 3f, g, h and Supplementary Figs. 13 15). Notably, we found TA-NPs could be effectively excited by the white light source with ultra-low power density (room light, $58 \mu \mathrm{W} / \mathrm{cm}^{2}$ ) and was able to exhibit bright afterglow (Fig. 3i). This ultra-bright afterglow luminescence of TA-NPs facilitated to shorten the signal acquisition time. Especially, when the acquisition time was reduced to $0.01 \mathrm{~s}$, TA-NPs could still produce strong afterglow luminescence. However, no obvious afterglow signals were detected from MEHPPV-NPs under the same conditions (Fig. $3 i$, Supplementary Fig. 16). Notably, the ultra-low power density $\left(58 \mu \mathrm{W} / \mathrm{cm}^{2}\right)$ that is far below the intensity of direct sunshine $\left(0.13 \mathrm{~W} / \mathrm{cm}^{2}\right)$ can provide safe excitation condition and thereby avoid photoinduced tissue damage.

Moreover, the ultra-low power of excitation light leads to the negligible chemical decomposition of afterglow molecules. Thereby, the afterglow signal could be recharged by more than 15 cycle light irradiation $\left(30 \mathrm{~mW} / \mathrm{cm}^{2}, 3 \mathrm{~s}\right)$ or 30 -cycle light irradiation $\left(6.6 \mathrm{~mW} / \mathrm{cm}^{2}, 1 \mathrm{~s}\right)$, with no obvious decay of maximum afterglow intensity (Fig. 3j, Supplementary Fig. 17). Such negligible afterglow photobleaching may be attributed to the efficient photo-afterglow conversion and ultra-low power density of excitation light, which enables to guarantee the accurate quantification of afterglow signals during hours-long and repeated molecular imaging. 
Prior to afterglow luminescence imaging in vivo, we studied the tissue penetration depth of afterglow luminescence emitted from TA-NPs. Although afterglow and fluorescence signals were declined with the increase of tissues thickness (Fig. 3k, Supplementary Fig. 18), the afterglow luminescence exerted significantly higher signal-to-background ratio (SBR) and tissue penetration (up to $6.0 \mathrm{~cm}$ ), compared with that of fluorescence, owing to the no autofluorescence from tissues during afterglow imaging. Besides, the tissue penetration and SBR of afterglow luminescence for TA-NPs was much higher than that for MEHPPV-NPs (Supplementary Fig. 19).

Two reasons may contribute to the significant improvement of afterglow intensity for TA-NPs, in contrast to MEHPPV-NPs. Firstly, for Type I afterglow, TA-NPs can generate $\mathrm{O}_{2}{ }^{-}$through the electron transfer and produce a large amount of EPOs via Type I pathway, while there was no obvious generation of $\mathrm{O}_{2}{ }^{--}$and a lack of Type I path for MEHPPV-NPs (Supplementary Fig. 20a). Secondly, for Type II afterglow, TA-NPs display the stronger ability to generate ${ }^{1} \mathrm{O}_{2}$, compared to MEHPPV-NPs (Supplementary Fig. 20b and c), which could promote the subsequent generation of EPOs via Type Il pathway. Thus, TA-NPs emitted an extremely high afterglow luminescence than MEHPPV-NPs, through both electron transfer path (Type I afterglow) and the enhanced energy transfer path (Type II afterglow) (Fig. 3e).

\section{Afterglow imaging of tumors and plaque-bearing carotid}

The features of TA-NPs, such as extremely high afterglow luminescence and prolonged luminescence lifetime, offer it noteworthy advantages in designing afterglow molecular imaging in vivo. Prior to afterglow luminescence imaging using TA-NPs in vivo, the stability and biocompatibility of TA-NPs were examined. The hydrodynamic size and luminescence intensity of TA-NPs remained highly stable after incubating those in different buffer solution (cell culture medium, PBS, water) for various time (Supplementary Figs. 7 and 21). Meanwhile, TA-NPs demonstrated no obvious adverse effect on cellular viability to CT-26 cells (mouse colon cancer cells) (Supplementary Fig. 22).

Inspired by the excellent afterglow performance of TA-NPs, we applied TA-NPs for afterglow luminescence imaging of subcutaneous CT-26 tumor-bearing mice after intratumoral injection, through IVIS Lumina XR imaging system (Fig. 4a). In dark condition, there was no afterglow signal at the tumor site at the beginning. Then, those mice were taken out of dark box and accepted the irradiation of smart mobile phone $\left(1.0 \mathrm{~mW} / \mathrm{cm}^{2}\right)$ or electric torch $\left(4.0 \mathrm{~mW} / \mathrm{cm}^{2}\right)$. The afterglow luminescence of mice was collected immediately after irradiation, and the tumor areas showed strong afterglow luminescence. Importantly, mice could be even excited by room light with ultra-low power density ( $\left.58 \mu \mathrm{W} / \mathrm{cm}^{2}\right)$ (Fig. $4 \mathrm{a}$ ). Moreover, the afterglow luminescence of tumor could well be recharged by multiple irradiation (more than 3 cycles) with electric torch, mobile phones or room light irradiation (Fig. 4b, Supplementary Fig. 23), as well as showed no obvious attenuation in maximum intensity during 15-cycle light irradiation (30 $\mathrm{mW} / \mathrm{cm}^{2}$ ) (Fig. 4c, Supplementary Fig. 23). The above data demonstrated TA-NPs in mice could be efficiently re-excited via low power density of portable light sources and displayed no notable afterglow photobleaching. 
Highly efficient afterglow luminescence of TA-NPs can significantly reduce the required dose for imaging in vivo. Under white light at low-power density of $6.6 \mathrm{~mW} / \mathrm{cm}^{2}$, TA-NPs (20x) showed afterglow signal as large as $\sim 6 \times 10^{7} \mathrm{p} / \mathrm{sec} / \mathrm{cm}^{2} / \mathrm{sr}$, which is significantly higher than that of MEHPPV-NPs (20x). When the lower dose $(1 \times)$ was used for TA-NPs, the signal intensity was still $\sim 4$ time that of MEHPPV-NPs $(20 x)$ (Fig. 4d, Supplementary Fig. 24). Afterward, we applied TA-NPs for afterglow luminescence imaging and fluorescence imaging of subcutaneous CT-26 tumors after intravenous injection. Afterglow and fluorescence images showed TA-NPs could effectively target tumor areas via enhanced permeability and retention (EPR) effect (Supplementary Fig. 25).

Tightly controlled behavior (mice in anesthetization) is more experimentally tractable, but it provides a limited view or information of the mice ${ }^{32}$. The ability to image a freely moving animal would represent a critical step towards understanding the relationship between the brain function and the established learning paradigms (fear conditioning, novel object recognition or social interactions), or the pathophysiology of conditions ${ }^{33-35}$. However, the real-time imaging of those unrestrained animals is greatly challenging. Mice movements would cause motion artifacts and distorted images, when the rapid movements of mice body were faster than the frame acquisition on a time scale, which is manageable in anesthetized animals but is exacerbated in awaken state ${ }^{36,37}$. Thereby, the visualizing study of freely moving animals requires the imaging system with ultra-high signal intensity of probe and ultra-short acquisition time. Fortunately, the Type I and Type II afterglow of TA-NPs guarantee extremely large luminescence intensity and ultra-short acquisition time $(0.01 \mathrm{~s})$, thereby we tried to investigate the applicability of TA-NPs for ultra-fast afterglow imaging of naturally behaving animals (Fig.

4e). Amazingly, TA-NPs allowed the observation of afterglow luminescence in a freely moving mouse for more than $10 \mathrm{~min}$, through subcutaneous injection (Fig. 4f, Supplementary Fig. 26). Especially, we were able to monitor the development of afterglow signal in subcutaneous CT-26 tumors in the freely moving mouse via intravenous injection of TA-NPs (Fig. 4g, Supplementary Fig. 28), while no afterglow signals were detected from MEHPPV-NPs -injected mice under same condition (acquisition time: $0.01 \mathrm{~s}$; irradiation conditions: $30 \mathrm{~mW} / \mathrm{cm}^{2}$ ) (Supplementary Figs. 27 and 29). By providing a moment-by-moment record of behavior, TA-NPs with high luminescence could create a serial of frameworks for relating natural behaviors to the afterglow signals, so as to assess sensitive behavioral changes in the freely moving animals.

Glioblastoma multiforme (GBM) is the most common and aggressive malignant tumor found in the central nervous system, with remarkably poor prognosis showing 5-year survival rate of 4 - $5 \%$ in clinic $^{38}$. Thereby, the precise imaging of GBM is beneficial to early diagnosis, and of great value in assessment of GBM aggressiveness, treatment response, prognosis and recurrence ${ }^{39-41}$. Owing to the non-invasion, high sensitivity and bright luminescence, we promoted TA-NPs for imaging orthotopic GBMbearing mice (Fig. 4h). The orthotopic GBM was prepared via implantation of human glioma cells (U87) into nude mice by stereotactic implantation and confirmed via hematoxylineosin (H\&E) staining of brain slice (Fig. 4k). After intravenous injection of TA-NPs, the orthotopic GBM-bearing mice showed the dynamically enhanced afterglow signals in head areas, while healthy mice displayed no obvious 
afterglow signals of mice head (Fig. 4i, j, Supplementary Fig. 30). Thus, TA-NPs could diffuse into the orthotopic glioma tumor via EPR effect through disrupted blood-brain barrier, and exerted high-sensitivity and accurate imaging of glioma tumor model.

The vascular system dysfunction due to atherosclerosis is one of leading causes for severe health problems around the world ${ }^{42,43}$. The plaque buildup in the walls of carotid arteries subsequently leads to luminal stenosis, low blood supply to brain, and even acute stroke incidents with plaque rupture ${ }^{44}$. Next, TA-NPs were applied to image the plaque in carotid atherosclerosis-bearing mice through intravenous injection of TA-NPs (Fig. 4l). From afterglow and fluorescence images of mice (Fig. 4m, n, Supplementary Figs. 31 - 32), the atherosclerotic plaque-bearing carotid (right side) showed significantly higher signal intensity than the normal carotid (left side). Moreover, ex vivo afterglow and fluorescence images displayed the plaque-bearing carotid with stronger signal than normal carotid (Fig. 40, Supplementary Fig. 33), which was consistent with the histological analysis of Oil red $O$ staining and H\&E staining (Supplementary Figs. 34 - 35). Those results suggested that TA-NPs were able to target atherosclerotic lesions and exert sensitive and non-invasive afterglow imaging of atherosclerotic plaque in living mice.

\section{Afterglow imaging of immune responses after chemotherapy in vivo}

Dynamic imaging of immune cell activity (cytotoxic $T$ lymphocytes ( $T$ cell) activity) during immunotherapy could enable to directly monitor immune response of tumor toward immunotherapy ${ }^{45}$. Granzyme $B$ is a serine-protease released by $C D 8^{+} T$ cells during the cellular immune response and represents one of the two dominant mechanisms by which T cells mediate cancer cell death ${ }^{46-50}$. To visualize granzyme B during immunotherapy, probe should possess the sufficient signal intensity, high imaging penetration depth, high SBR and no obvious afterglow photobleaching, which allows for accurate quantification over the repeated imaging. Encouraged by the excellent afterglow performance of TA-NPs, we further promoted TA-NPs for afterglow imaging cytotoxic T lymphocytes activity within tumors after chemotherapy or radiotherapy. To achieve this, we designed, synthesized and characterized a functional activity-based Granzyme B afterglow nanoprobe (TA-BHQ), where a Granzyme B-cleavable peptide sequence (lle-Glu-Phe-Asp, IEFD) was conjugated with TA-NPs as luminophores and BHQ-3 as quenchers (Fig. 5a; Supplementary Methods: Preparation of TA-BHQ and Supplementary Fig. 36). These donor-acceptor pairs with excellent spectral overlap resulted in the maximum efficacy of quenching luminescence ("off" state) through afterglow resonance energy transfer (ARET), when spatially constrained (less than $10 \mathrm{~nm}$ ) (Fig. 5b). In the presence of Granzyme B, the IEFD peptide between TA-NPs and BHQ-3 was cleaved and ARET was reduced, leading to the recovery of afterglow luminescence ("on" state) (Fig. 5a). As a result, the gradual increase in afterglow intensity was observed over concentrations of Granzyme B (Fig. 5c). Signal quantification from afterglow or fluorescent images showed a linear correlation between luminescence intensities and Granzyme B concentrations (Fig. 5d, Supplementary Fig. 37). Besides, the luminescence signals remained nearly unchanged for other enzymes and biomolecules, demonstrating the selective response of TA-NPs probes toward Granzyme B (Supplementary Fig. 38). 
The capabilities of TA-BHQ probes for in vivo real-time afterglow luminescence imaging of Granzyme B were validated against subcutaneous CT-26 tumor-bearing mice model. To activate the immune response, three immunotherapeutic agents including NLG919, BMS-1 and BEC were administered into the living mice (Fig. 5e, Supplementary Fig. 39). These immunotherapeutic agents mainly acted in tumor microenvironment to facilitate the infiltration of $\mathrm{CD}^{+} \mathrm{T}$ cells into tumor. Using BEC-treated mice as an example, the afterglow and fluorescence signals of tumors were increased gradually after injection of TABHQ (Supplementary Fig. 40). At 6 h post-injection of TA-BHQ, strong afterglow signals were observed for the tumor of immunotherapeutic-treated mice, which was higher than that of PBS group. Moreover, multiple-time injection of BEC showed stronger afterglow signals than that of one-time injection, indicating the dose-dependent immune response (Fig. $5 f, g$ ). Although the fluorescence imaging of Granzyme B via TA-BHQ were consistent with that of those afterglow imaging, the SBR of afterglow was higher than that of fluorescence (Supplementary Figs. 41 - 42).

Next, immunofluorescence staining and flow cytometry analysis were performed for measuring $\mathrm{CD} 8^{+} \mathrm{T}$ cells infiltration and Granzyme B level within tumors after various treatments (Fig. 5h, Supplementary Figs. 43 - 45). Those tumor tissues excised from BEC-treated mice displayed higher infiltration of $\mathrm{CD} 8^{+} \mathrm{T}$ cells and higher expression of Granzyme B, as compared to other groups, which revealed a higher level of immunoactivation after BEC-treatment. As summarized in heat map (Fig. 5i), the ex vivo quantitative analyses of immunofluorescence staining were coincided with the signals of afterglow, which validated that the activated signals of TA-BHQ were well correlated with the immunoactivation.

\section{Afterglow imaging of radiotherapy or/and immune checkpoint blockade therapy}

Radiotherapy (RT), referring to the external-beam X-ray irradiation, has gained acceptance for treating over $50 \%$ of cancer patients in clinic ${ }^{51,52}$. Ionizing radiation can trigger immune system response through multiple ways, such as augmenting the expression of MHC-I in the affected cells, attenuating PD$1 /$ CTLA-4 in T lymphocytes, or inducing immunogenic cell death ${ }^{53,54}$. Thereby, there is a growing consensus that the combination of immune checkpoint inhibitors (such as anti-PD-L1) with RT can increase immune response rates ${ }^{55,56}$. Unfortunately, studies have also provided the intriguing outcomes of immune response to different doses and fractionation schedules of $\mathrm{RT}^{54}$.

Considering the lack of validated tools for predicting the immune response to RT or/and immune checkpoint blockade therapy, we favored to promote TA-BHQ for evaluating the immune response in mice receiving RT combined with anti-PD-L1, by monitoring Granzyme B level. The subcutaneous CT-26 tumorbearing mice received RT or/and anti-PD-L1 treatment, followed by injection of TA-BHQ and afterglow luminescence imaging (Fig. 6a). Notably, mice treated with anti-PD-L1 + RT showed dynamic enhancement of afterglow signals from tumor sites post injection TA-BHQ (Fig. 6b, c, Supplementary Fig. 46). Specifically, compared with mice treated with PBS, those tumors treated with anti-PD-L1, RT or antiPD-L1 + RT exhibited higher afterglow signals (Fig. 6d, e), which indicated TA-BHQ could monitor Granzyme B level during RT or/and immune checkpoint blockade therapy. Besides, those fluorescence imaging results were well matched with that of afterglow imaging (Supplementary Fig. 47). 
Next, the expression level of Granzyme B and population of T cell in tumors were measured by immunofluorescence staining and flow cytometry analysis (Fig. 6f, g, h, Supplementary Fig. 48). Those results demonstrated that the combination treatment (anti-PD-L1 + RT) led to more infiltration of CD8 ${ }^{+} T$ cells and higher level of Granzyme B in subcutaneous CT-26 tumor, compared with single treatment (RT or anti-PD-L1) or PBS treatment, which validated that the activation of TA-BHQ probe at tumor site was owing to the increased level of Granzyme B released from activated CD8 ${ }^{+} T$ cells infiltrated in the tumors. Moreover, the heat map (Fig. 6i) displayed the afterglow signals were well correlated with both $\mathrm{CD}^{+} \mathrm{T}$ cells infiltration and Granzyme B levels within tumors after various treatments. Therefore, TA-BHQ was able to assess the real-time immunoactivation in living mice during chemotherapy, RT or/and immune checkpoint blockade therapy.

\section{Conclusion}

In summary, we have designed trianthracene derivatives (TA)-based nanoparticles as a new type of afterglow luminescence nanoprobe for high-sensitive, safe, accurate and dynamic imaging. TA-NPs emitted an extremely high afterglow luminescence than MEHPPV-NPs, through both electron transfer path (Type I afterglow) and the enhanced energy transfer path (Type II afterglow). Excited by light irradiation with ultra-low power density, TA-based nanoparticles showed no obvious attenuation of maximum intensity in mice even after 15-cycle excitation. Such ultra-bright afterglow and negligible photobleaching endowed TA-NPs to realize rechargeable, accurate and longitudinal imaging of subcutaneous tumor, orthotopic glioma and plaque-bearing carotid. Notably, the ultra-high afterglow signals of TA-based nanoparticles were captured under as short as $0.01 \mathrm{~s}$ of acquisition time, resulting in their ability for ultra-fast afterglow imaging of unrestrained subcutaneous tumors-bearing mice, and thereby the potential for gaining further insight into pathophysiology mechanism of neurologic diseases (such as epilepsy, stroke, Alzheimer disease, Parkinson). Furthermore, the engineering of TA-NPs facilitates the development of smart activatable afterglow nanoprobe (TA-BHQ) to directly monitor the efficacy of immunotherapies by imaging Granzyme B. Specifically, the afterglow luminescence of TA-BHQ were activated by Granzyme B during chemotherapy, radiotherapy or/and immune checkpoint blockade therapy, enabling the dynamic and accurate imaging of $T$ cell-based immunotherapies in mice. In the future, TA-BHQ probe may be extended to evaluate the immune activation of distant tumors, or even estimate the role of RT dose/ fractionation toward immunomodulation, so as to guide the individualized immunotherapy.

\section{Declarations}

\section{Acknowledgments}

This work was supported by the National Key R\&D Program of China (2019YFA0210103), National Natural Science Foundation of China (grants 51872088, 21804039, 21977027, 21675043, 21890744, and 91959104), and the Fundamental Research Funds for the Central Universities. 


\section{Author contributions}

G.S. and X.Z. designed the research. Y.W., J.G. and S.L. designed and completed the test experiment in solution. L.S. designed and built the cytotoxicity experiment. Y.W., S.L., L.X. and Q.C. designed and built the animal experiment for molecular imaging and therapy. Y.W. designed the schematic diagram. Y.W., G.S., X.Z., and W.T. analyzed and interpreted the data and wrote the manuscript.

\section{Competing interests}

The authors declare that they have no other competing interests.

\section{References}

1. Meade, T.J. \& Aime, S. Chemistry of Molecular Imaging. Acc. Chem. Res. 42, 821-821 (2009).

2. Achilefu, S. Introduction to Concepts and Strategies for Molecular Imaging. Chem. Rev. 110, 25752578 (2010).

3. Huang, X. et al. Ratiometric optical nanoprobes enable accurate molecular detection and imaging. Chem. Soc. Rev. 47, 2873-2920 (2018).

4. Wang, T. et al. A hybrid erbium(III)-bacteriochlorin near-infrared probe for multiplexed biomedical imaging. Nat. Mater. 20, 1571-1578 (2021).

5. Xu, Z. et al. A Visible and Near-Infrared, Dual-Channel Fluorescence-On Probe for Selectively Tracking Mitochondrial Glutathione. Chem 4, 1609-1628 (2018).

6. Li, B. et al. Organic NIR-II molecule with long blood half-life for in vivo dynamic vascular imaging. Nat. Commun. 11, 3102 (2020).

7. Zhu, S. et al. Molecular imaging of biological systems with a clickable dye in the broad 800- to 1,700nm near-infrared window. PNAS114, 962 (2017).

8. Chen, Z.Y. et al. Advance of molecular imaging technology and targeted imaging agent in imaging and therapy. BioMed Res. Int. 2014, 819324 (2014).

9. Atreya, R. \& Goetz, M. Molecular imaging in gastroenterology. Nat. Rev. Gastroenterol. Hepatol. 10, 704-712 (2013).

10. Wu, L. et al. Forster resonance energy transfer (FRET)-based small-molecule sensors and imaging agents. Chem. Soc. Rev. 49, 5110-5139 (2020).

11. Yang, Q. et al. Rational Design of Molecular Fluorophores for Biological Imaging in the NIR-II Window. Adv. Mater. 29 (2017).

12. Smith, B.R. \& Gambhir, S.S. Nanomaterials for In Vivo Imaging. Chem. Rev. 117, 901-986 (2017).

13. Miao, Q. et al. Molecular afterglow imaging with bright, biodegradable polymer nanoparticles. Nat. Biotechnol. 35, 1102-1110 (2017).

14. Wu, L. et al. H2S-activatable near-infrared afterglow luminescent probes for sensitive molecular imaging in vivo. Nat. Commun. 11, 446 (2020). 
15. Maldiney, T. et al. The in vivo activation of persistent nanophosphors for optical imaging of vascularization, tumours and grafted cells. Nat. Mater. 13, 418-426 (2014).

16. Pei, P. et al. X-ray-activated persistent luminescence nanomaterials for NIR-II imaging. Nat. Nanotechnol. 16, 1011-1018 (2021).

17. Jiang, Y. \& Pu, K. Molecular Probes for Autofluorescence-Free Optical Imaging. Chem. Rev. (2021) DOI: 10.1021/acs.chemrev.1c00506.

18. Jiang, Y. \& Pu, K. Multimodal Biophotonics of Semiconducting Polymer Nanoparticles. Acc. Chem. Res. 51, 1840-1849 (2018).

19. Li, J. \& Pu, K. Development of organic semiconducting materials for deep-tissue optical imaging, phototherapy and photoactivation. Chem. Soc. Rev. 48, 38-71 (2019).

20. Li, Y., Gecevicius, M. \& Qiu, J. Long persistent phosphors-from fundamentals to applications. Chem. Soc. Rev. 45, 2090-2136 (2016).

21. Sun, S.K., Wang, H.F. \& Yan, X.P. Engineering Persistent Luminescence Nanoparticles for Biological Applications: From Biosensing/Bioimaging to Theranostics. Acc. Chem. Res. 51, 1131-1143 (2018).

22. Cui, D., Xie, C., Li, J., Lyu, Y. \& Pu, K. Semiconducting Photosensitizer-Incorporated Copolymers as Near-Infrared Afterglow Nanoagents for Tumor Imaging. Adv. Healthcare Mater. 7, e1800329 (2018).

23. Xie, C., Zhen, X., Miao, Q., Lyu, Y. \& Pu, K. Self-Assembled Semiconducting Polymer Nanoparticles for Ultrasensitive Near-Infrared Afterglow Imaging of Metastatic Tumors. Adv. Mater. 30, e1801331 (2018).

24. Jiang, Y. et al. A generic approach towards afterglow luminescent nanoparticles for ultrasensitive in vivo imaging. Nat. Commun. 10, 2064 (2019).

25. Ni, X. et al. Near-Infrared Afterglow Luminescent Aggregation-Induced Emission Dots with Ultrahigh Tumor-to-Liver Signal Ratio for Promoted Image-Guided Cancer Surgery. Nano Lett. 19, 318-330 (2019).

26. $\mathrm{Xu}, \mathrm{Y}$. et al. An aggregation-induced emission dye-powered afterglow luminogen for tumor imaging. Chem. Sci. 11, 419-428 (2020).

27. Tian, Q. et al. Hydrophilic flower-like CuS superstructures as an efficient $980 \mathrm{~nm}$ laser-driven photothermal agent for ablation of cancer cells. Adv. Mater. 23, 3542-3547 (2011).

28. Skylaki, S., Hilsenbeck, O. \& Schroeder, T. Challenges in long-term imaging and quantification of single-cell dynamics. Nat. Biotechnol. 34, 1137-1144 (2016).

29. Magidson, V. \& Khodjakov, A. Circumventing photodamage in live-cell microscopy. Method. Cell Biol. $114,545-560$ (2013).

30. Fudickar, W. \& Linker, T. Why triple bonds protect acenes from oxidation and decomposition. J. Am. Chem. Soc. 134, 15071-15082 (2012).

31. He, Y.Q. et al. Capture and Release of Singlet Oxygen in Coordination-Driven Self-Assembled Organoplatinum(II) Metallacycles. J. Am. Chem. Soc. 142, 2601-2608 (2020).

32. Chen, X. et al. Sensory evoked fMRI paradigms in awake mice. Neuroimage 204, 116242 (2020). 
33. Iwano, S. et al. Single-cell bioluminescence imaging of deep tissue in freely moving animals. Science 359, 935-939 (2018).

34. Lee, J.U. et al. Non-contact long-range magnetic stimulation of mechanosensitive ion channels in freely moving animals. Nat. Mater. 20, 1029-1036 (2021).

35. Stowers, J.R. et al. Virtual reality for freely moving animals. Nat. Methods 14, 995-1002 (2017).

36. Helmchen, F., Fee, M.S., Tank, D.W. \& Denk, W. A Miniature Head-Mounted Two-Photon Microscope: High-Resolution Brain Imaging in Freely Moving Animals. Neuron 31, 903-912 (2001).

37. Kyme, A.Z. et al. Open-field PET: Simultaneous brain functional imaging and behavioural response measurements in freely moving small animals. Neuroimage 188, 92-101 (2019).

38. Batash, R., Asna, N., Schaffer, P., Francis, N. \& Schaffer, M. Glioblastoma Multiforme, Diagnosis and Treatment; Recent Literature Review. Curr. Med. Chem. 24, 3002-3009 (2017).

39. Song, G. et al. Carbon-coated FeCo nanoparticles as sensitive magnetic-particle-imaging tracers with photothermal and magnetothermal properties. Nat. Biomed. Eng. 4, 325-334 (2020).

40. Song, G. et al. A Magneto-Optical Nanoplatform for Multimodality Imaging of Tumors in Mice. ACS Nano 13, 7750-7758 (2019).

41. Kasten, B.B. et al. Targeting MMP-14 for dual PET and fluorescence imaging of glioma in preclinical models. Eur. J. Nucl. Med. Mol. Imaging 47, 1412-1426 (2020).

42. Beaglehole, R. \& Yach, D. Globalisation and the prevention and control of non-communicable disease: the neglected chronic diseases of adults. Lancet 362, 903-908 (2003).

43. Song, P. et al. Global and regional prevalence, burden, and risk factors for carotid atherosclerosis: a systematic review, meta-analysis, and modelling study. Lancet Global Health 8, e721-e729 (2020).

44. Libby, P. et al. Atherosclerosis. Nat. Rev. Dis. Primers 5, 56 (2019).

45. Zhong, Y. et al. In vivo molecular imaging for immunotherapy using ultra-bright near-infrared-llb rareearth nanoparticles. Nat. Biotechnol. 37, 1322-1331 (2019).

46. Mac, Q.D. et al. Non-invasive early detection of acute transplant rejection via nanosensors of granzyme B activity. Nat. Biomed. Eng. 3, 281-291 (2019).

47. Nguyen, A. et al. Granzyme B nanoreporter for early monitoring of tumor response to immunotherapy. Sci. Adv. 6, eabc2777 (2020).

48. He, S., Li, J., Lyu, Y., Huang, J. \& Pu, K. Near-Infrared Fluorescent Macromolecular Reporters for RealTime Imaging and Urinalysis of Cancer Immunotherapy. J. Am. Chem. Soc. 142, 7075-7082 (2020).

49. Scott, J.I. et al. A Functional Chemiluminescent Probe for in Vivo Imaging of Natural Killer Cell Activity Against Tumours. Angew. Chem. Int. Ed. 60, 5699-5703 (2021).

50. Zhang, Y. et al. Activatable Polymeric Nanoprobe for Near-Infrared Fluorescence and Photoacoustic Imaging of T Lymphocytes. Angew. Chem. Int. Ed. 60, 5921-5927 (2021).

51. Chen, X., Song, J., Chen, X. \& Yang, H. X-ray-activated nanosystems for theranostic applications. Chem. Soc. Rev. 48, 3073-3101 (2019). 
52. Zheng, X. et al. A Near-Infrared Phosphorescent Nanoprobe Enables Quantitative, Longitudinal Imaging of Tumor Hypoxia Dynamics during Radiotherapy. Cancer Res. 79, 4787-4797 (2019).

53. Chen, Q., Chen, M. \& Liu, Z. Local biomaterials-assisted cancer immunotherapy to trigger systemic antitumor responses. Chem. Soc. Rev. 48, 5506-5526 (2019).

54. Mortezaee, K. \& Najafi, M. Immune system in cancer radiotherapy: Resistance mechanisms and therapy perspectives. Crit. Rev. Oncol. Hematol. 157, 103180 (2021).

55. Chen, J.L. et al. Evaluation of antitumor immunity by a combination treatment of high-dose irradiation, anti-PDL1, and anti-angiogenic therapy in murine lung tumors. Cancer Immunol. Immunother. 70, 391-404 (2021).

56. Pouessel, D. et al. Hypofractionnated stereotactic radiotherapy and anti-PDL1 durvalumab combination in recurrent glioblastoma: Results of the phase I part of the phase I/II STERIMGLI trial. J. Clin. Oncol. 36, 2046-2046 (2018).

\section{Table 1}

Table 1 is available in the Supplementary Files section.

\section{Figures}


a

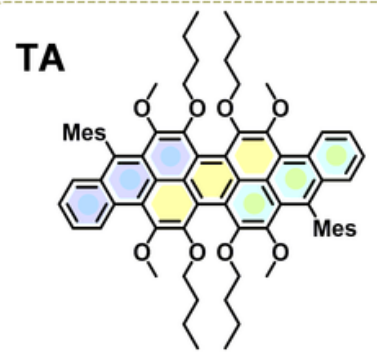

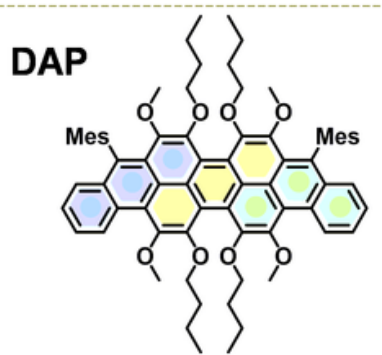

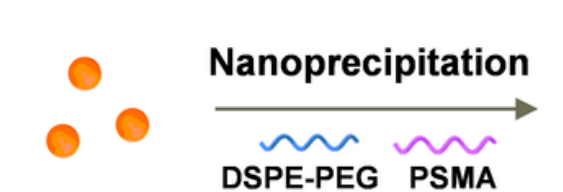

Afterglow molecules

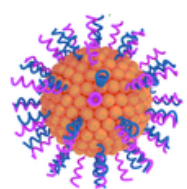

Afterglow nanoparticles

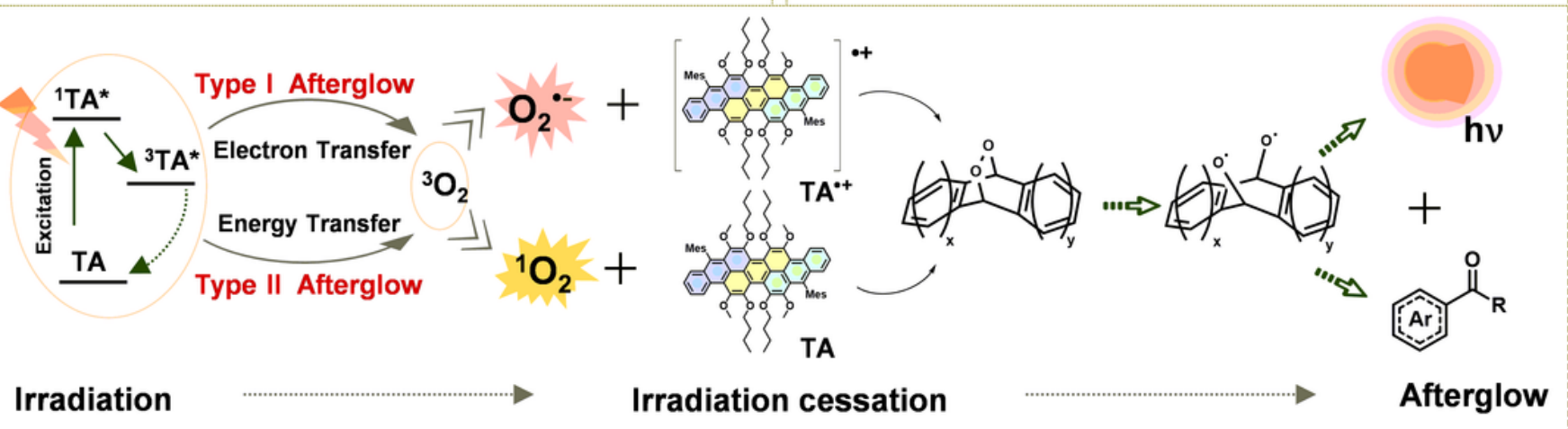

b
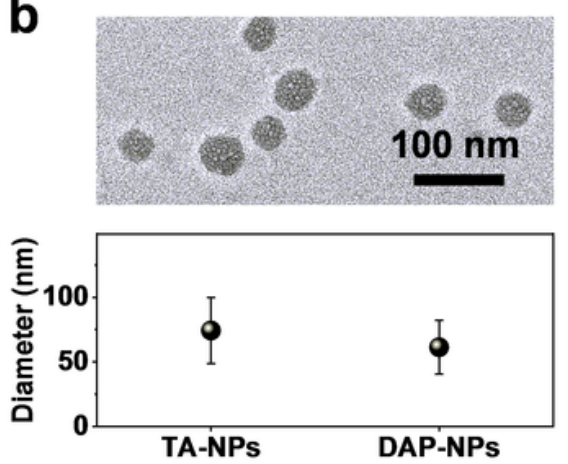

e

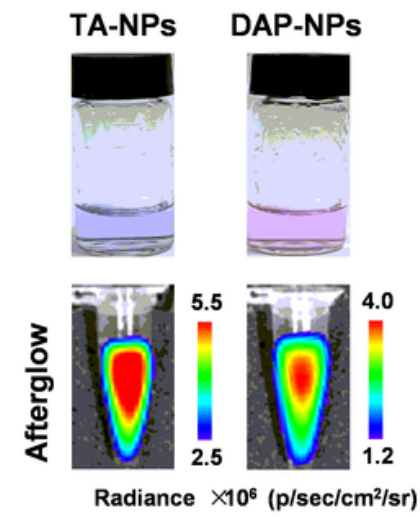

C

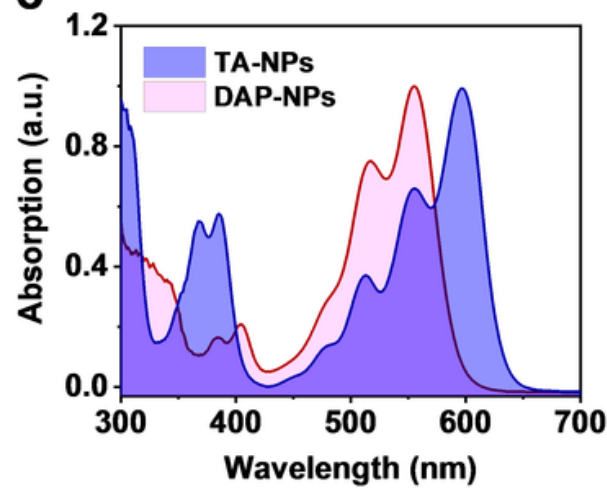

f

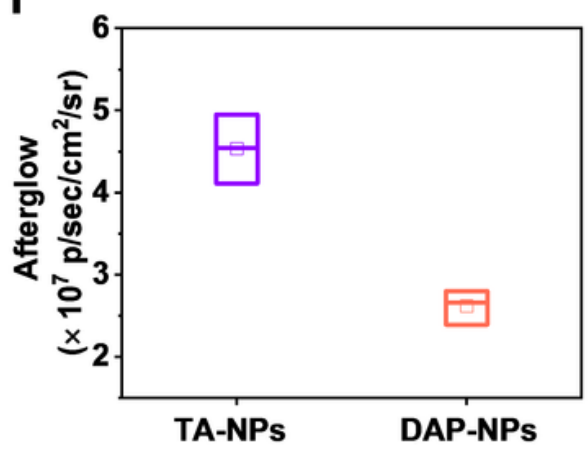

d

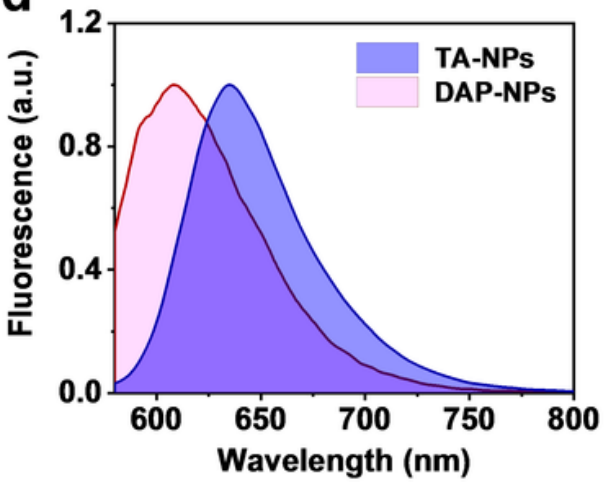

g

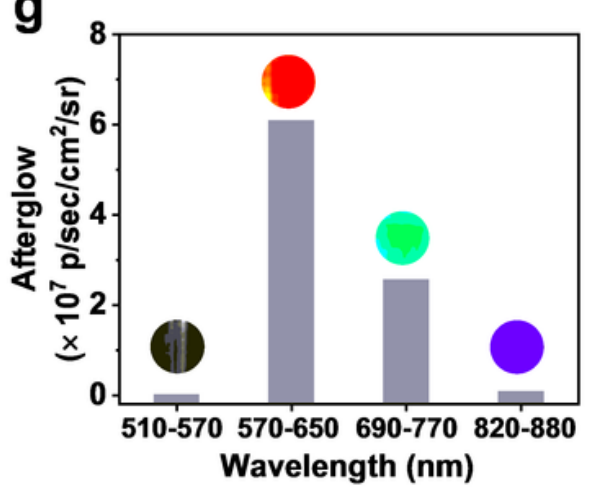

Figure 1

Synthesis and characterization of afterglow nanoparticles (TA-NPs and DAP-NPs). (a) Chemical structures of afterglow molecules, the illustration for preparation of afterglow nanoparticles, and scheme for afterglow luminescent mechanisms of TA-NPs. R were alkyl chains. (b) The representative TEM image of TA-NPs, and average hydrodynamic diameters of TA-NPs and DAP-NPs in H2O. (c) UV-vis absorption spectra of TA-NPs and DAP-NPs in H2O. (d) Fluorescence spectra of TA-NPs and DAP-NPs in H2O. (e) Photographs (upper panel), and afterglow images (lower panel) of TA-NPs and DAP-NPs. (f) 
Quantification of afterglow intensities of TA-NPs and DAP-NPs in (e). (g) Afterglow emission of TA-NPs, obtained through different channels.

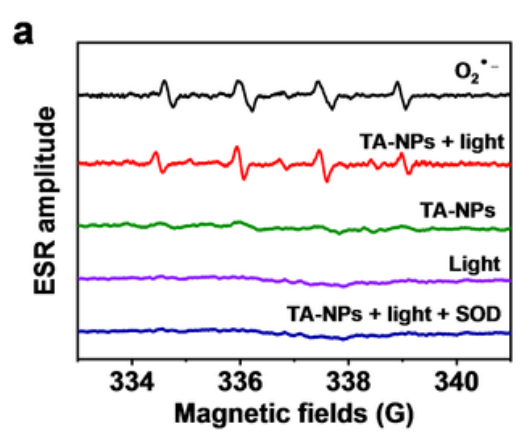

d

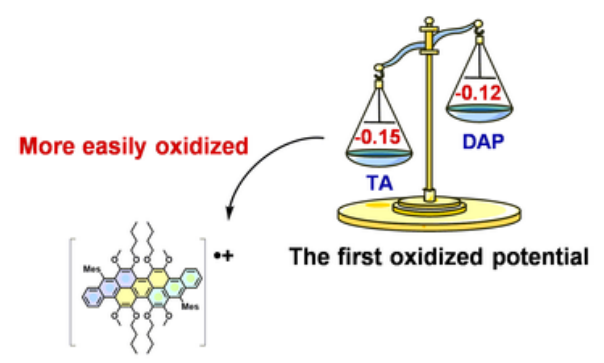

b

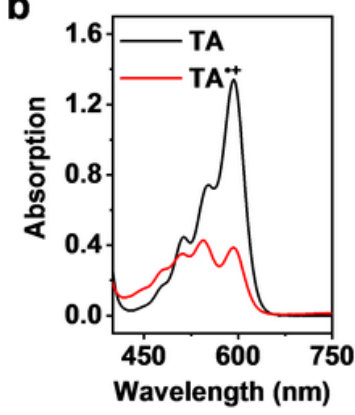

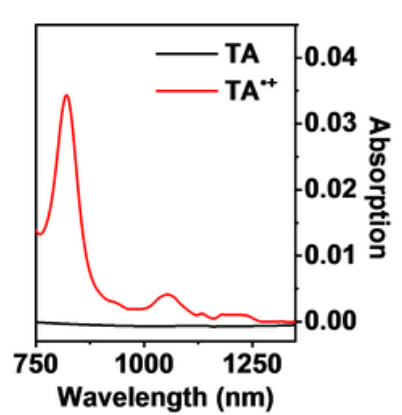

C

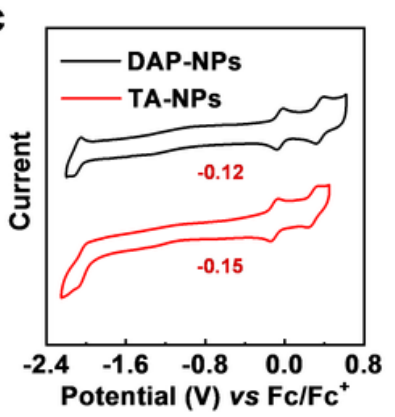

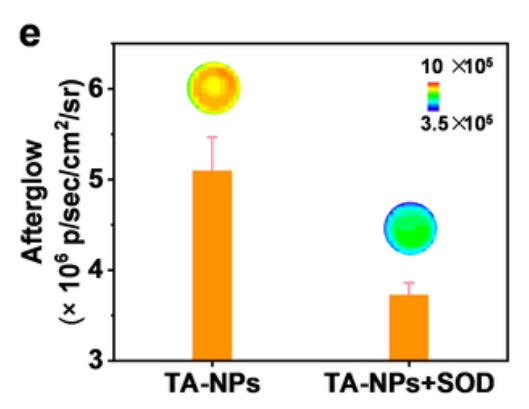
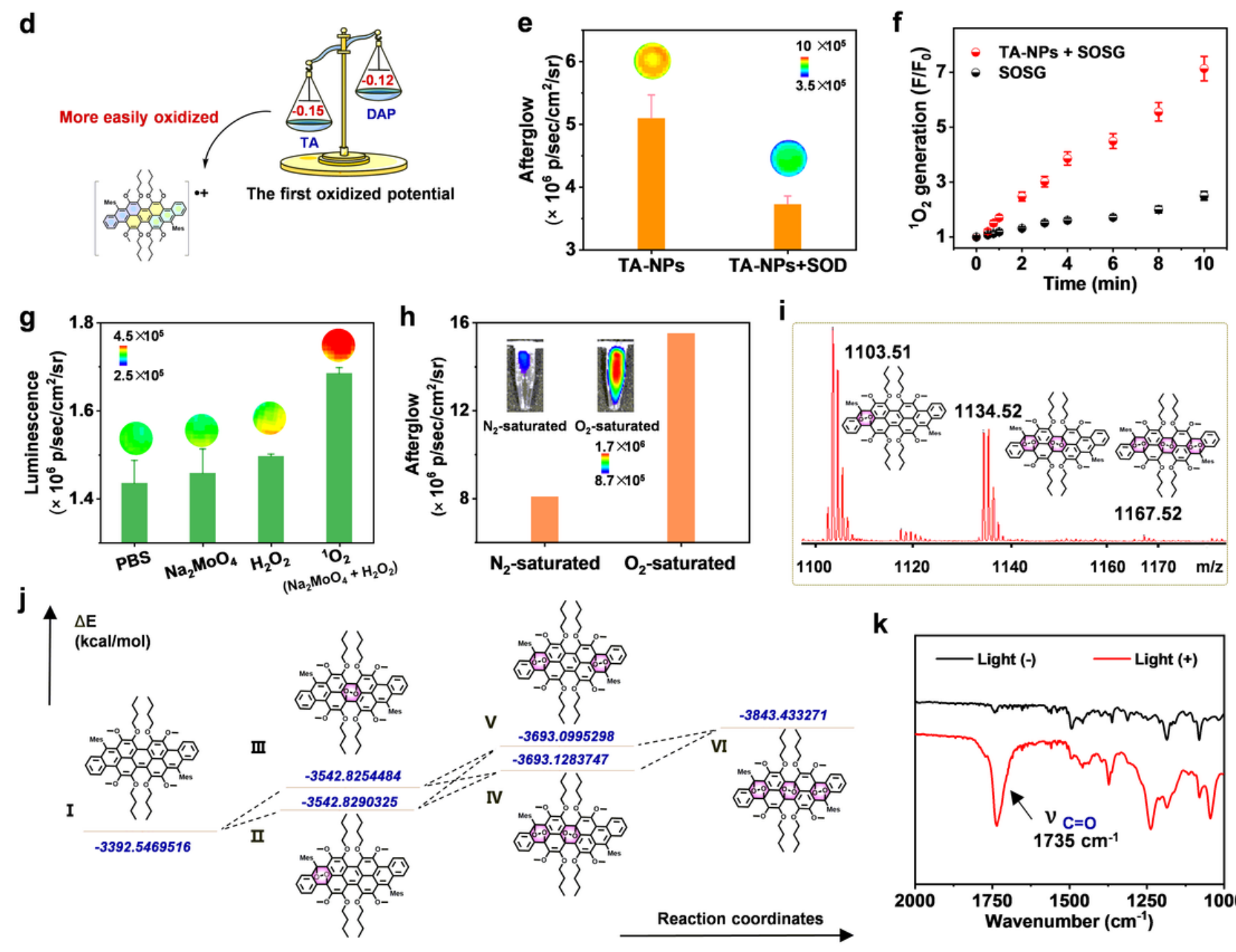

k

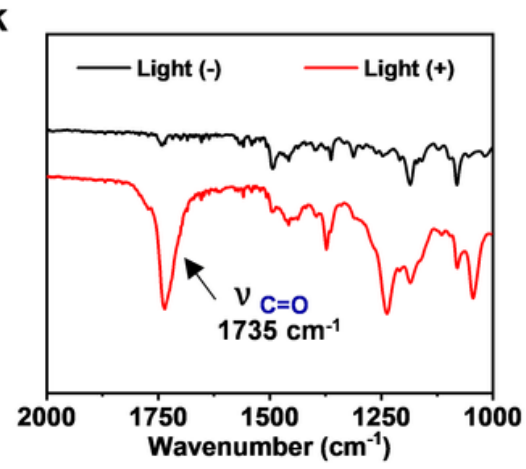

\section{Figure 2}

Mechanism for afterglow luminescence of TA-NPs. (a) ESR signals of DMPO for 02-- characterization, KO2 as the reference. (b) UV-vis-NIR absorption spectra of TA and corresponding TA + recorded in DCM solution. (c) Cyclic voltammograms of TA (red curves) and DAP (black curves) in DCM solution (1.0 mM). (d) Scheme for possibility of oxidation reaction of TA and DAP with 02. (e) Afterglow intensities of TANPs incubated with H2O or SOD. (f) 102 generation of TA-NPs as a function of light irradiation time. 102 generation was determined by fluorescence enhancement (F/FO) of SOSG at $530 \mathrm{~nm}$. (g) Luminescent 
intensities of TA-NPs incubated with PBS, Na2MoO4, H2O2, or Na2MoO4 + H2O2. (h) Afterglow intensities of TA-NPs under 02-saturated or N2-saturated conditions. (i) MALDI-TOF-MS spectrum of EPOs complexes at $1 \mathrm{~h}$ after light irradiation of TA molecule. (j) DFT calculations for the formation pathways of EPOs from TA molecules. (k) FTIR spectra of TA in THF receiving light irradiation (30 $\mathrm{mW} / \mathrm{cm} 2$ ) for $24 \mathrm{~h}$ or not.

a
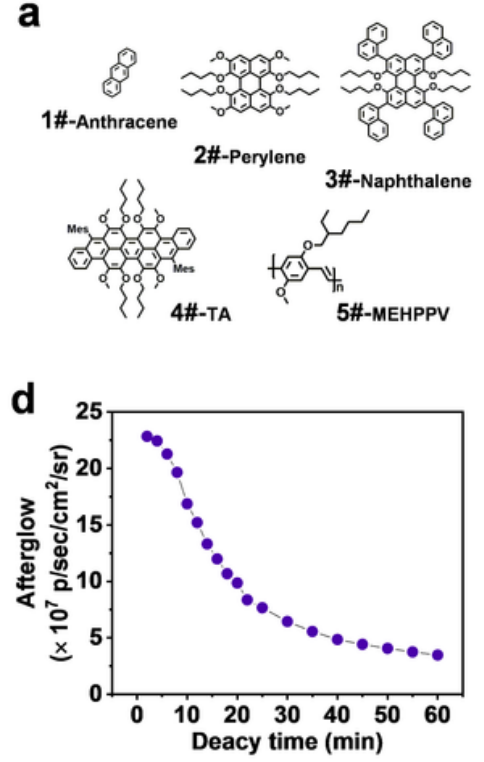

g

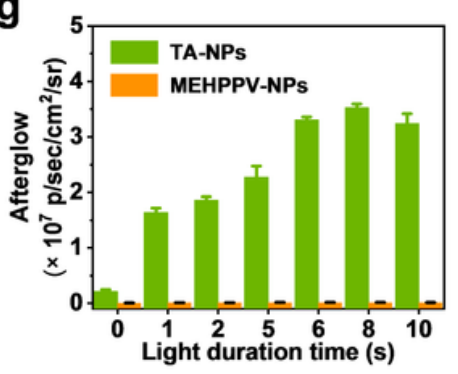

h

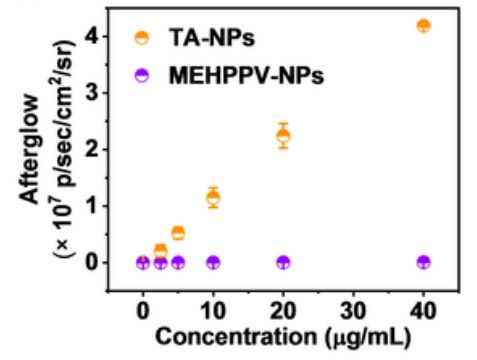

b

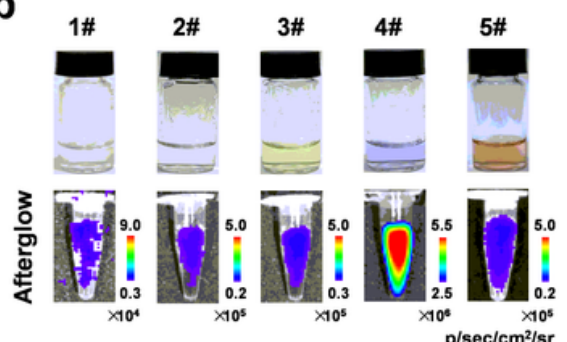

C

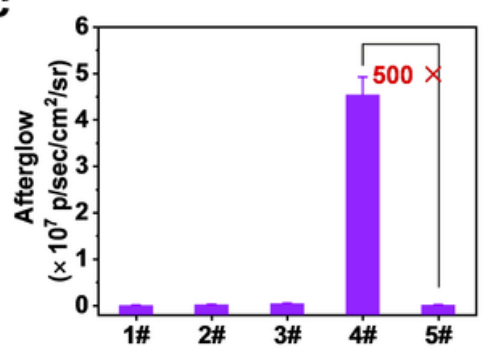

e

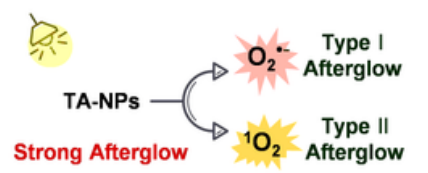

f

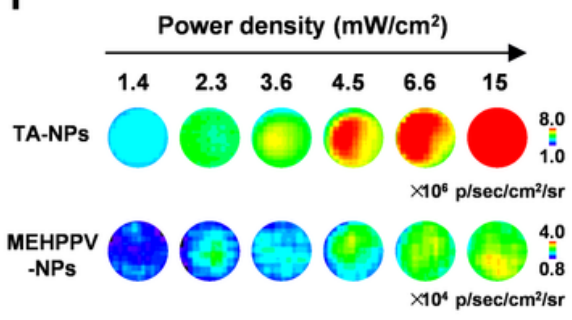

i
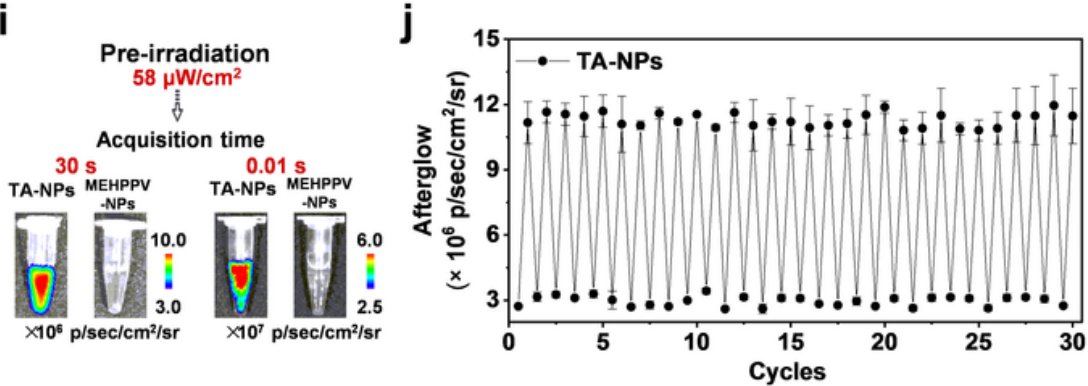

k The thickness of chicken tissue (cm)

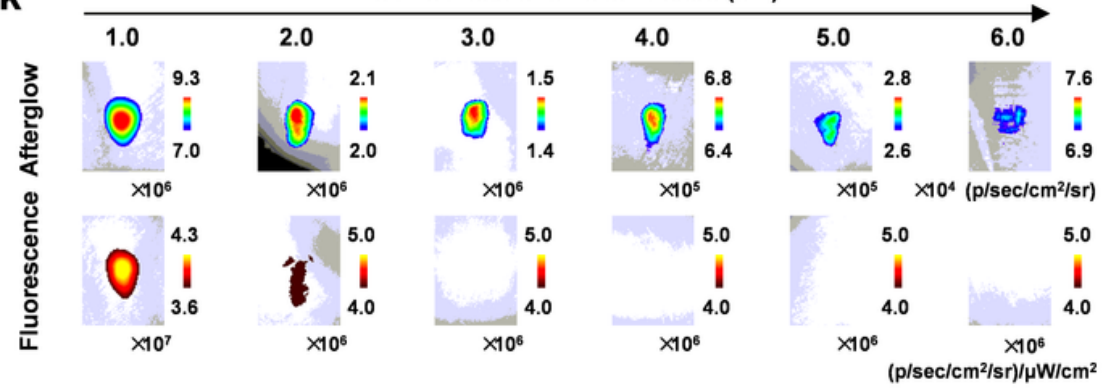

\section{Figure 3}

Luminescent properties of afterglow nanoparticles. (a) Chemical structures of afterglow molecules. 1\#, Anthracene; 2\#, Perylene (Per-4OMe); 3\#, Naphthalene (Per-4n); 4\#, TA; 5\#, MEHPPV. (b) Photographs (upper panel), and afterglow images (lower panel) of those nanoparticles. (c) Quantification of afterglow intensities in (b). (d) The decay of afterglow intensity of TA-NPs after irradiation. (e) Schematic illustration of the improvement of afterglow intensity for TA-NPs through Type I and II afterglow reaction, in contrast to MEHPPV-NPs. (f) The afterglow images of TA-NPs and MEHPPV-NPs under light irradiation 
of different power density. (g) Afterglow intensities of TA-NPs and MEHPPV-NPs after receiving light irradiation of different time. (h) Afterglow intensities of different concentrations of TA-NPs and MEHPPVNPs. (i) The afterglow images of TA-NPs and MEHPPV-NPs under room light irradiation $(58 \mu \mathrm{W} / \mathrm{cm} 2)$ with $30 \mathrm{~s}$ or $0.01 \mathrm{~s}$ acquisition time. (j) Afterglow intensities of TA-NPs as a function of cycles of light irradiation (6.6 mW/cm2). (k) Afterglow images (upper panel) and fluorescence images (lower panel) of TA-NPs through tissues of different thickness.
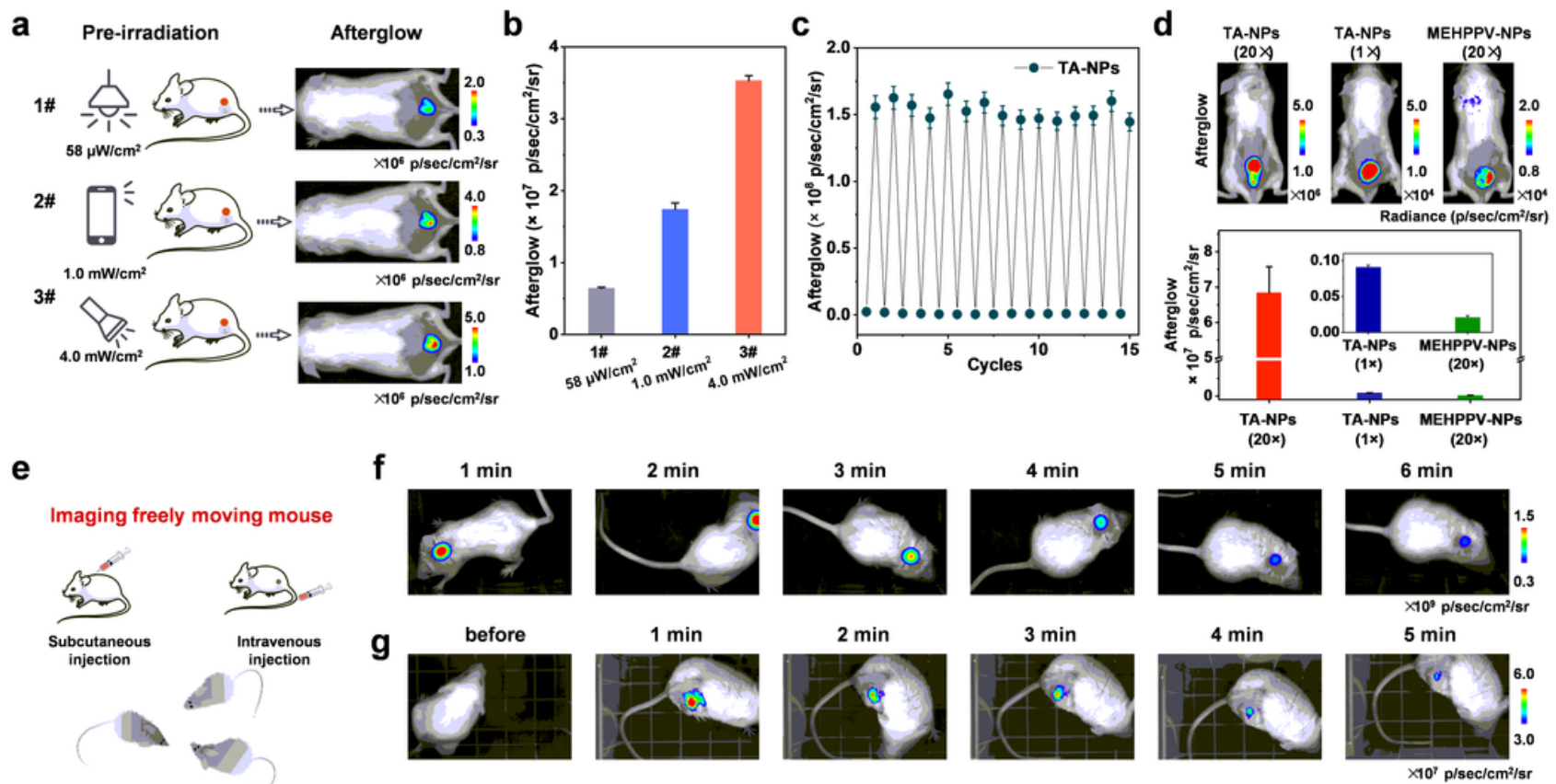

h

Imaging of orthotopic glioma
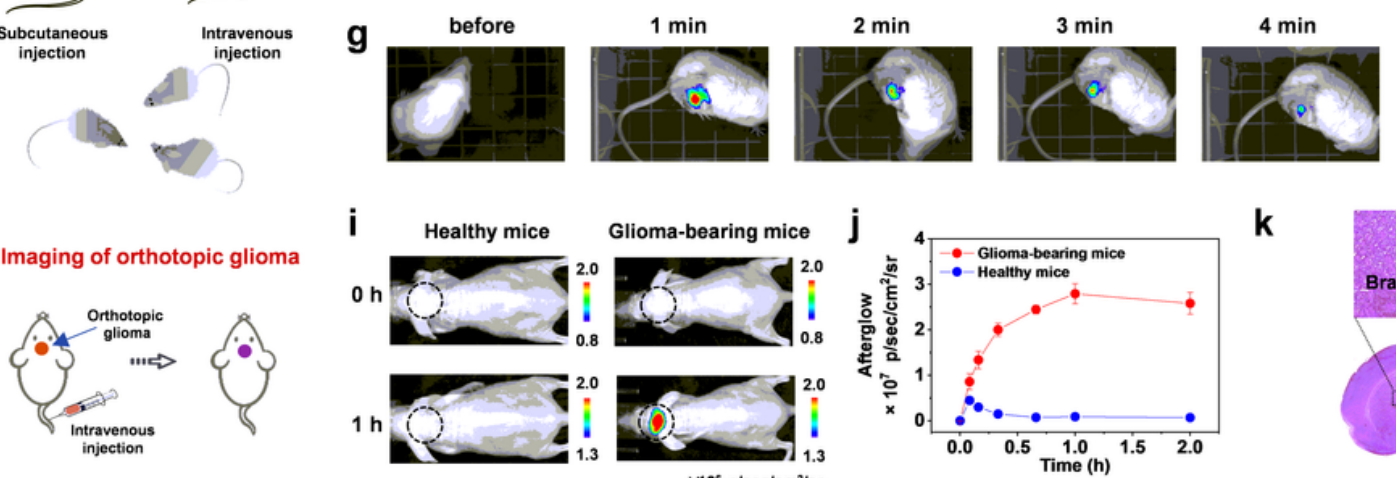

5 min

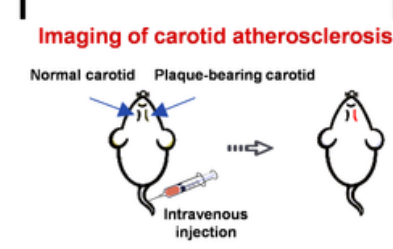

m
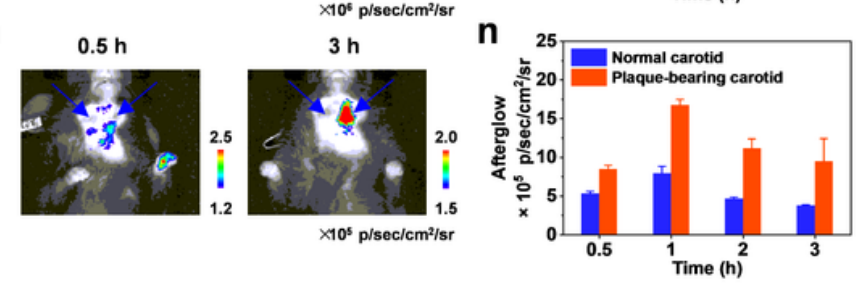

k
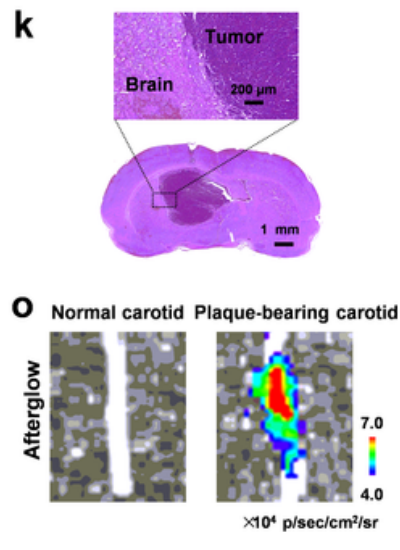

\section{Figure 4}

In vivo afterglow imaging of tumors and plaque-bearing carotid. (a - b) Subcutaneous CT-26 tumorsbearing mice were i.t injected with TA-NPs and pre-irradiated by white light sources with different power density (room light, $58 \mu \mathrm{W} / \mathrm{cm} 2$; smart phone light, $1.0 \mathrm{~mW} / \mathrm{cm} 2$; electric torch light, $4.0 \mathrm{~mW} / \mathrm{cm} 2$ ). (a) Schematic illustration. (b) Quantification of afterglow intensities for tumor areas in (a). (c) Afterglow intensities of tumor injected with TA-NPs as a function of cycle of light irradiations $(30 \mathrm{~mW} / \mathrm{cm} 2)$. (d) Subcutaneous CT-26 tumors-bearing mice were i.t. injected with TA-NPs (20x), TA-NPs (1x) or MEHPPVNPs (20x), respectively. Afterglow images of those mice and corresponding intensities from tumor sites. 
(e) Schematic illustration of afterglow imaging in freely moving mice. (f) Afterglow images of freely moving mice at different time points post subcutaneous injection of TA-NPs. (h) Afterglow images of freely moving mice bearing subcutaneous CT-26 tumors, at different time post i.v. injection of TA-NPs. (h) Schematic illustration for glioma-bearing mice i.v. injected with TA-NPs and afterglow imaging. (i) Afterglow images of healthy mice or glioma-bearing mice at different points post injection. (j) The afterglow intensities of mice head from healthy mice or glioma-bearing mice. (k) H\&E staining images of brain slice indicating the existing glioma. ( $\mathrm{I}$ - o) Mice with normal carotid (left side) and plaque-bearing carotid (right side) were i.v. injected with TA-NPs and followed by afterglow imaging. (I) Schematic illustration. ( $m$ ) Afterglow images of mice at different points post injection. ( $n$ ) The afterglow intensities of normal carotid or plaque-bearing carotid. (o) Afterglow images of the excised normal carotid or plaquebearing carotid ex vivo.

a
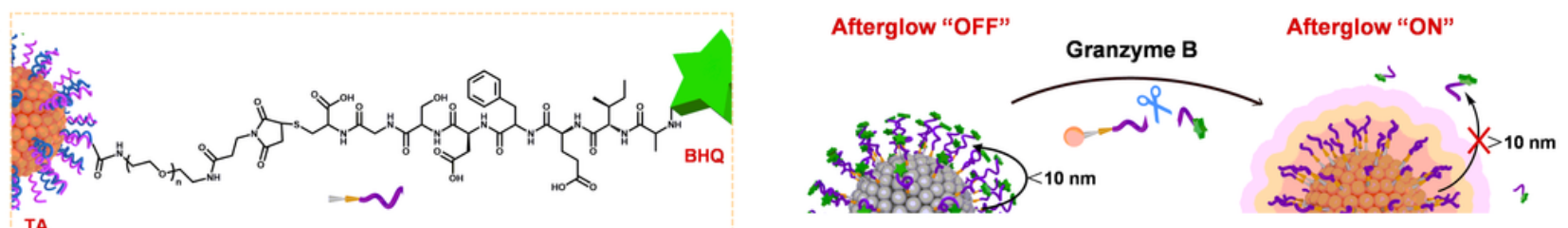

TA-NPS-PEG-CGSDFEIA-BHQ

Afterglow resonance energy transfer

Absence of resonance energy transfer

b

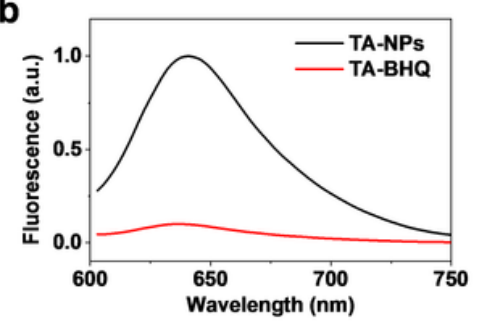

c

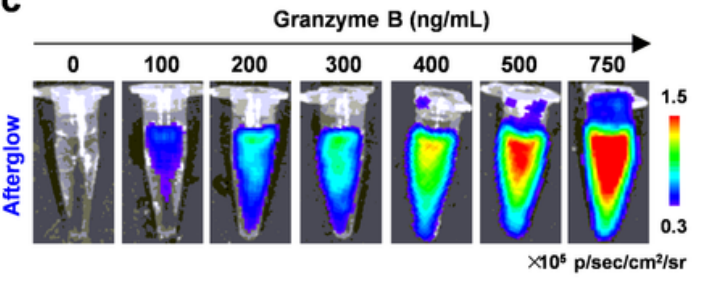

d

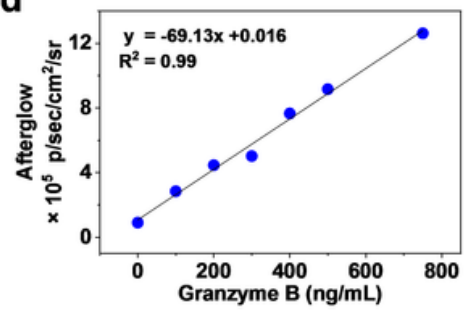

e
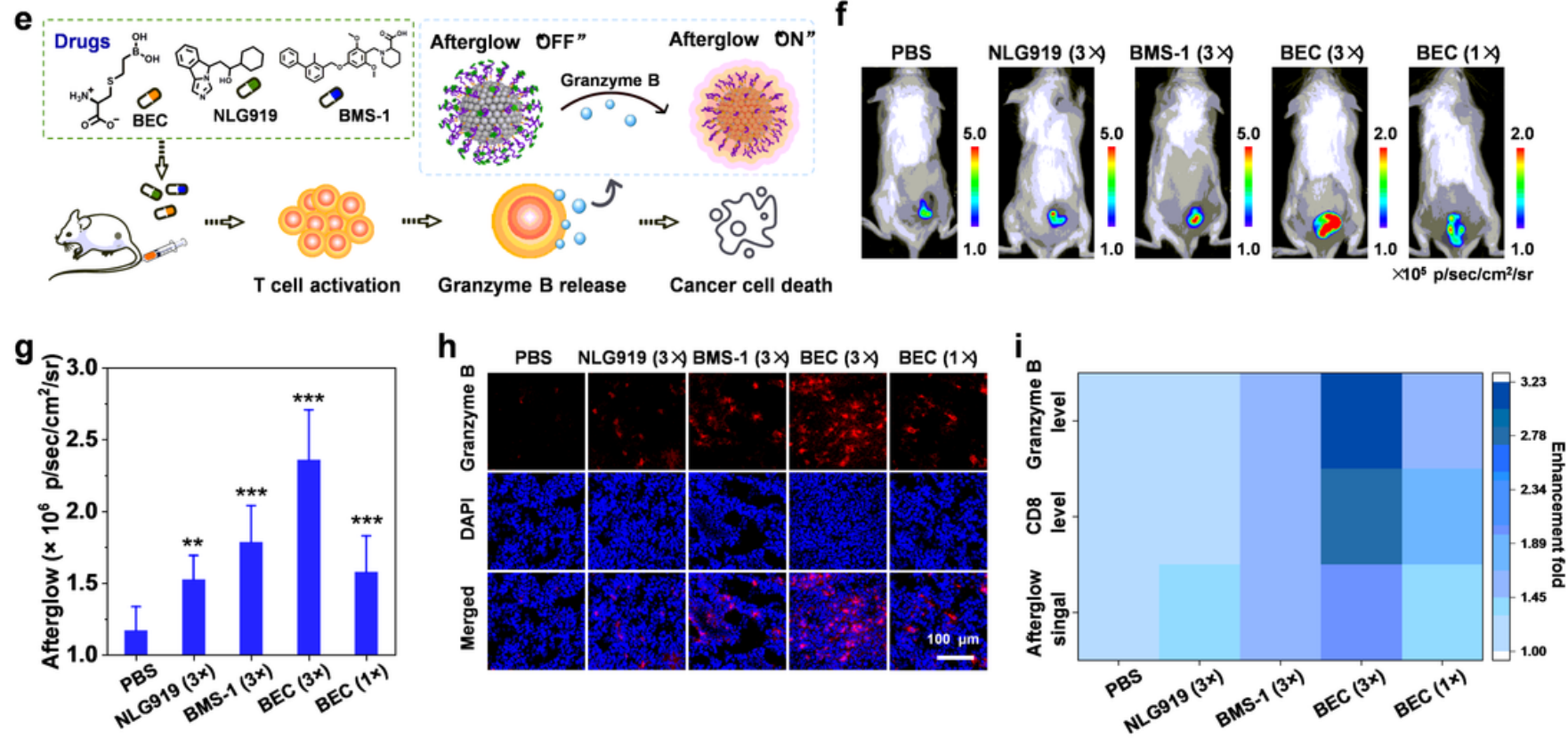

Figure 5 
Afterglow imaging of immune responses after chemotherapy in vivo. (a) Scheme of TA-BHQ nanoprobe for afterglow luminescence imaging Granzyme B. (b) Fluorescence spectra of TA-NP and TA-BHQ under the same concentration of TA. (c) Afterglow images of TA-BHQ incubated with various concentrations of Granzyme B. (d) Plot of afterglow intensity as the concentration of Granzyme B. (e - i) Subcutaneous CT26 tumor-bearing mice were i.v. injected with (I) PBS, (II) NLG919 (3x), (III) BMS-1 (3x), (IV) BEC (3x); (V) BEC (1x), respectively, then injected with TA-NPs, followed by afterglow imaging. (e) Schematic illustration of TA-BHQ for imaging of Granzyme B after treatment. (f) Representative afterglow images of mice from each group at $6 \mathrm{~h}$ post injection of TA-BHQ. (g) Corresponding afterglow intensities from tumor areas in (f). (h) Confocal fluorescence images of tumor slices stained with Granzyme B antibody (Red color), from each group. (i) Heat map showed the correlation between afterglow signals of TA-BHQ, Granzyme B expression levels, and CD8+ T cells within tumor tissues for each group. 
a

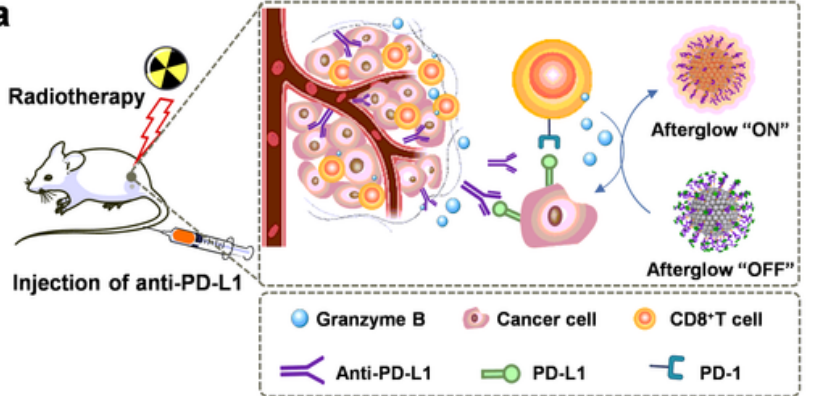

b
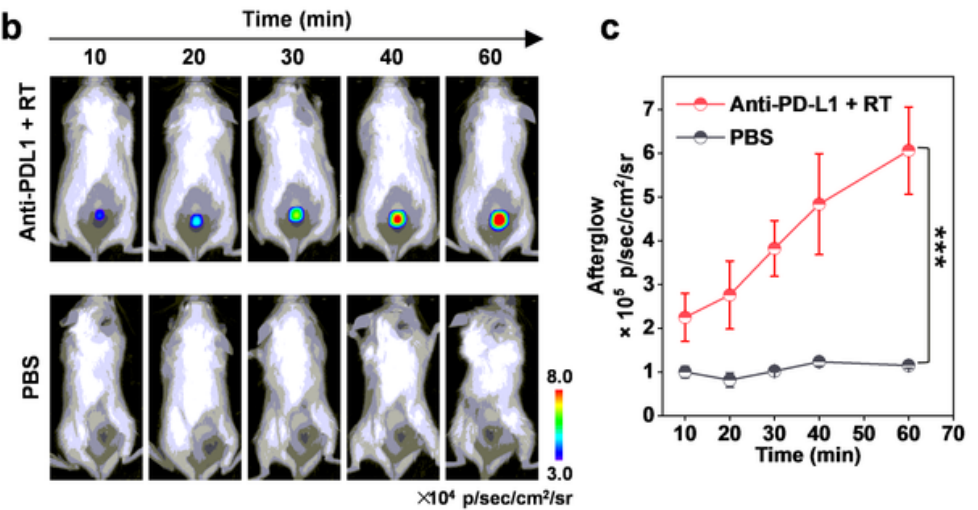

f

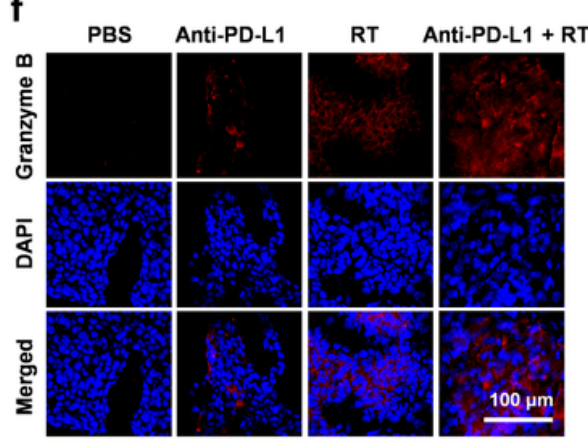

h

i
Radiation therapy Anti-PD-L1 TA-NPs In vivo imaging Ex vivo analysis

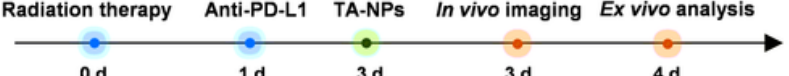

PBS
Anti-PD-L1
RT
Anti-PD-L1
+ RT

- $m \Leftrightarrow$ Tumor collection

Flow cytometry analysis Immunofluorescence staining

d
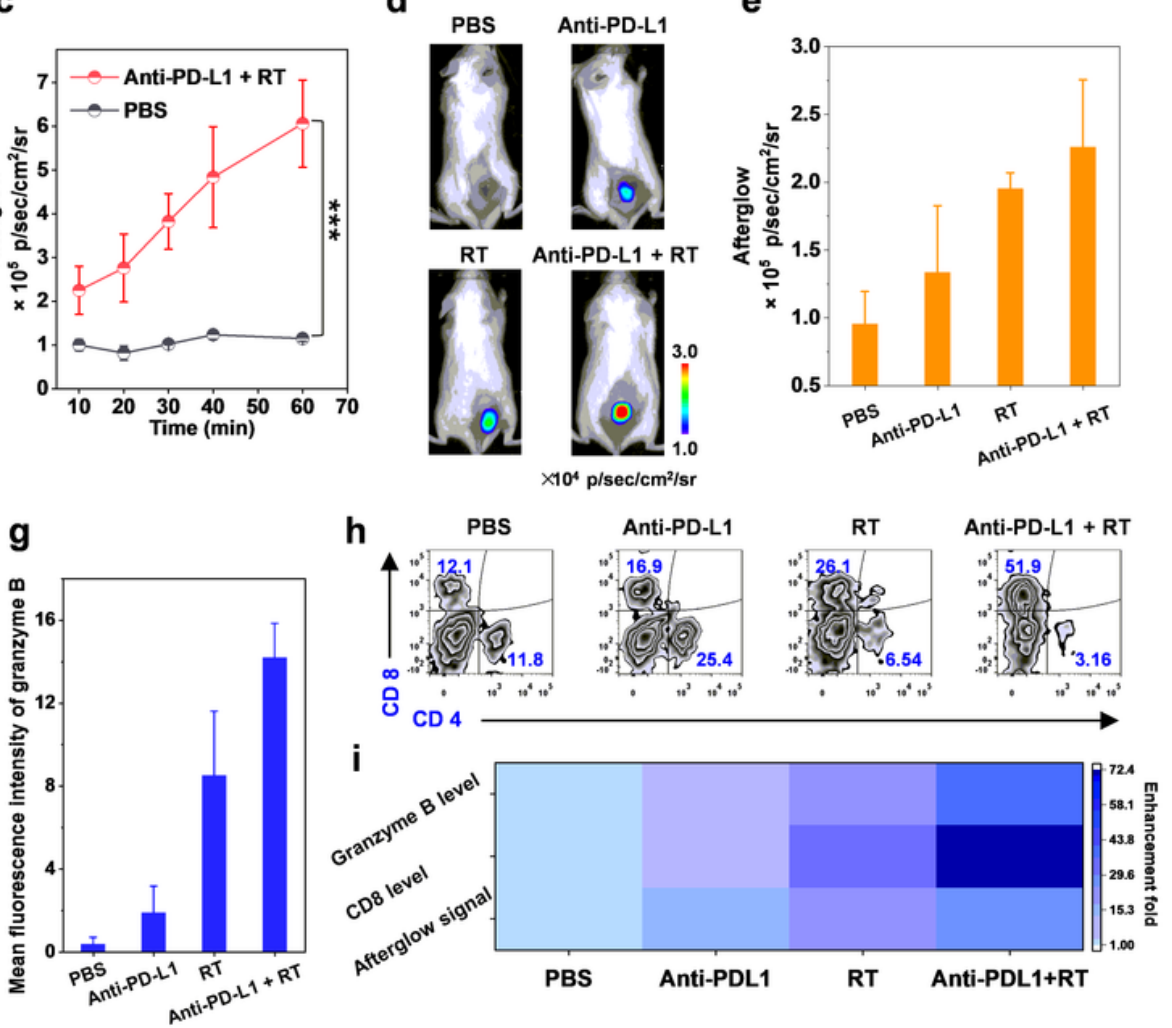

\section{Figure 6}

Afterglow imaging of radiotherapy or/and immune checkpoint blockade therapy in vivo. CT-26 tumorbearing mice received the following treatment: (I) PBS; (II) X-ray irradiation; (III) anti-PD-L1; (IV) X-ray irradiation + anti-PD-L1. There days later, those mice were injected with TA-NPs, followed by afterglow imaging. (a) Schematic illustration for TA-BHQ for imaging of Granzyme B after treatment and the detailed administration procedures. (b) Afterglow images of mice for (I) PBS and (IV) X-ray irradiation + anti-PD-L1 as a function of time. (c) The corresponding afterglow intensities from tumor sites in (b). (d) Representative afterglow images from four groups at 10 min post injection of TA-BHQ. (e) The 
corresponding afterglow intensities from tumor sites in (d). (f) Representative confocal fluorescence images of tumor slices stained with Granzyme B antibody (Red color), from each group. (g) Quantification of Granzyme B levels from confocal fluorescence images in (f). (h) Flow cytometry of cytotoxic T lymphocytes (CD8+ and CD4+) cells within tumor tissues from each group. (i) Heat map indicating the relationship of afterglow signals of TA-BHQ, Granzyme B expression levels, and CD8+ T cells within tumor tissues from each group.

\section{Supplementary Files}

This is a list of supplementary files associated with this preprint. Click to download.

- SupportingInformation.docx

- Table1.docx 